\title{
On the structure of Milnor $K$-groups of certain complete discrete valuation fields
}

\author{
par MASATO KURIHARA
}

\begin{abstract}
RÉSUMÉ. Pour un exemple typique de corps de valuation discrète complet $K$ de type II au sens de [12], nous déterminons les quotients gradués $\operatorname{gr}^{i} K_{2}(K)$ pour tout $i>0$. Dans l'appendice, nous décrivons les $K$-groupes de Milnor d'un certain anneau local à l'aide de modules de différentielles, qui sont liés à la théorie de la cohomologie syntomique.
\end{abstract}

ABSTRACT. For a typical example of a complete discrete valuation field $K$ of type II in the sense of [12], we determine the graded quotients $\operatorname{gr}^{i} K_{2}(K)$ for all $i>0$. In the Appendix, we describe the Milnor $K$-groups of a certain local ring by using differential modules, which are related to the theory of syntomic cohomology.

\section{Introduction}

In the arithmetic of higher dimensional local fields, the Milnor $K$-theory plays an important role. For example, in local class field theory of Kato and Parshin, the Galois group of the maximal abelian extension is described by the Milnor $K$-group, and the information on the ramification is in the Milnor $K$-group, at least for abelian extensions. So it is very important to know the structure of the Milnor $K$-groups.

Let $K$ be a complete discrete valuation field, $v_{K}$ the normalized additive valuation of $K, O_{K}$ the ring of integers, $m_{K}$ the maximal ideal of $O_{K}$, and $F$ the residue field. For $q>0$, the Milnor $K$-group $K_{q}^{M}(K)$ has a natural filtration $U^{i} K_{q}^{M}(K)$ which is by definition the subgroup generated by $\left\{1+m_{K}^{i}, K^{\times}, \ldots, K^{\times}\right\}$for all $i \geq 0$ (cf. $\S 1$ ). We are interested in the graded quotients $\operatorname{gr}^{i} K_{q}^{M}(K)=U^{i} K_{q}^{M}(K) / U^{i+1} K_{q}^{M}(K)$. The structures of $\operatorname{gr}^{i}$ were determined in Bloch [1] and Graham [5] in the case that $K$ is of equal characteristic. But in the case that $K$ is of mixed characteristics, much less is known on the structures of $\operatorname{gr}^{i} K_{q}^{M}(K)$. They are determined by Bloch and Kato [2] in the range that $0 \leq i \leq e_{K} p /(p-1)$ where $e_{K}=v_{K}(p)$ is the absolute ramification index. They are also determined in the case $e_{K}=v_{K}(p)=1$ (and $p>2$ ), in [14] for all $i>0$. This result was generalized in J. Nakamura [17] to the case that $K$ is absolutely tamely 
ramified (cf. also [16] where some special totally ramified case was dealt). We also remark that I. Zhukov calculated the Milnor $K$-groups of some higher dimensional local fields from a different point of view ([24]).

On the other hand, we encountered strange phenomena in [12] for certain $K$ (if $K$ is of type II in the terminology of [12]). Namely, if $K$ is of type II, for some $q$ we have $\operatorname{gr}^{i} K_{q}^{M}(K)=0$ for some $i$ (even in the case [F : $\left.\left.F^{p}\right]=p^{q-1}\right)$, which never happens in the equal characteristic case. A typical example of a complete discrete valuation field of type II is $K=K_{0}(\sqrt[p]{p T})$ where $K_{0}$ is the fraction field of the completion of the localization of $\mathbf{Z}_{p}[T]$ at the prime ideal $(p)$. The aim of this article is to determine all $\mathrm{gr}^{i} K_{2}^{M}(K)$ for this typical example of type II (Theorem 1.1), and to give a direct consequence of the theorem on the abelian extensions (Corollary 1.3). (For the structure of the $p$-adic completion of $K_{2}^{M}(K)$, see also Corollary 1.4).

I would like to thank K.Kato and Jinya Nakamura. The main result of this article is an answer to their question. I would also like to thank I.B.Fesenko for his interest in my old results on the Milnor $K$-groups of complete discrete valuation fields. This paper was prepared during my stay at University of Nottingham in 1996. I would like to express my sincere gratitude to their hospitality, and to the support from $\operatorname{EPSRC}(\mathrm{GR} / \mathrm{L} 06560)$. Finally, I would like to thank B. Erez for his constant efforts to edit the papers gathered on the occasion of the Luminy conference $\left(^{*}\right)$.

\section{Notation}

For an abelian group $A$ and an integer $n$, the cokernel (resp. kernel) of the multiplication by $n$ is denoted by $A / n$ (resp. $A[n]$ ), and the torsion subgroup of $A$ is denoted by $A_{\text {tors }}$. For a commutative ring $R, R^{\times}$denotes the group of the units in $R$. For a discrete valuation field $K$, the ring of integers is denoted by $O_{K}$, and the unit group of $O_{K}$ is denoted by $U_{K}$. For a Galois module $M$ and an integer $r \in \mathbf{Z}, M(r)$ means the Tate twist. We fix an odd prime number $p$ throughout this paper.

\section{Statement of the result}

Let $K_{0}$ be a complete discrete valuation field with residue field $F$. We assume that $K_{0}$ is of characteristic 0 and $F$ is of characteristic $p>0$, and that $p$ is a prime element of the integer ring $O_{K_{0}}$ of $K_{0}$. We further assume that $\left[F: F^{p}\right]=p$ and $p$ is odd.

We denote by $\Omega_{F}^{1}$ the module of absolute Kähler differentials $\Omega_{F / \mathbf{Z}}^{1}$. For a positive integer $n$, we define the subgroups $B_{n} \Omega_{F}^{1}$ by $B_{1} \Omega_{F}^{1}=d F \subset \Omega_{F}^{1}$ and $C^{-1} B_{n} \Omega_{F}^{1}=B_{n+1} \Omega_{F}^{1} / B_{1} \Omega_{F}^{1}$ for $n>0$ where $C^{-1}$ is the inverse Cartier operator (cf. [6] 0.2). Then $B_{n} \Omega_{F}^{1}$ gives an increasing filtration on $\Omega_{F}^{1}$. 
We fix a $p$-base $t$ of $F$, namely $F=F^{p}(t)$. (Recall that we are assuming $\left[F: F^{p}\right]=p$.) We take a lifting $T \in U_{K_{0}}$ of the $p$-base $t$ of $F$, and define $K=K_{0}(\sqrt[p]{p T})$. This is a discrete valuation field of type II in the sense of $[12]$.

In this article, we study the structure of $K_{2}(K)=K_{2}^{M}(K)$. As usual, we denote the symbol by $\{a, b\}$ (which is the class of $a \otimes b$ in $K_{2}(K)=$ $K^{\times} \otimes K^{\times} / J$ where $J$ is the subgroup generated by $a \otimes(1-a)$ for $a \in$ $\left.K^{\times} \backslash\{1\}\right)$. We write the composition of $K_{2}(K)$ additively. For $i>0$, we define $U^{i} K_{2}(K)$ to be the subgroup of $K_{2}(K)$ generated by $\left\{U_{K}^{i}, K^{\times}\right\}$ where $U_{K}^{i}=1+m_{K}^{i}$. We are interested in the graded quotients

$$
\operatorname{gr}^{i} K_{2}(K)=U^{i} K_{2}(K) / U^{i+1} K_{2}(K) .
$$

We also use a slightly different subgroup $\mathcal{U}^{i} K_{2}(K)$ which is, by definition, the subgroup generated by $\left\{U_{K}^{i}, U_{K}\right\}$. We have

$$
U^{1} K_{2}(K)=\mathcal{U}^{1} K_{2}(K) \supset U^{2} K_{2}(K) \supset \mathcal{U}^{2} K_{2}(K) \supset U^{3} K_{2}(K) \supset \ldots
$$

It is known that $K_{2}(K) / U^{1} K_{2}(K) \simeq F^{\times} \oplus K_{2}(F)$. Further, by Bloch and Kato [2] (cf. Remark 1.2), $\operatorname{gr}^{i} K_{2}(K)$ is determined in the range $1 \leq$ $i \leq p+1$ in our case (note that $e_{K}=v_{K}(p)=p$ ). In this article, we prove

Theorem 1.1. We put $\pi=\sqrt[p]{p T}$ which is a prime element of $O_{K}$.

(1) If $i>p+1$ and $i$ is prime to $p$, we have $\operatorname{gr}^{i} K_{2}(K)=0$.

(2) For $i=2 p$, we have $\mathcal{U}^{2 p} K_{2}(K) \subset U^{2 p+1} K_{2}(K)$, and the homomorphism $x \mapsto$ the class of $\left\{1+p \pi^{p} \widetilde{x}, \pi\right\}$

$$
F \longrightarrow \mathrm{gr}^{2 p} K_{2}(K)
$$

$\left(\widetilde{x}\right.$ is a lifting of $x$ to $O_{K}$ ) induces an isomorphism

$$
F / F^{p} \stackrel{\simeq}{\longrightarrow} \operatorname{gr}^{2 p} K_{2}(K) .
$$

(3) For $i=n p$ such that $n \geq 3$, we have $\mathcal{U}^{n p} K_{2}(K) \subset U^{n p+1} K_{2}(K)$, and the homomorphism $x \mapsto$ the class of $\left\{1+p^{n} \widetilde{x}, \pi\right\}$ ( $\widetilde{x}$ is a lifting of $x$ to $O_{K}$ ) gives an isomorphism

$$
F^{p^{n-2}} \stackrel{\simeq}{\longrightarrow} \mathrm{gr}^{n p} K_{2}(K) .
$$

Remark 1.2. We recall results of Bloch and Kato [2]. Let $K$ be a complete discrete valuation field of mixed characteristics $(0, p)$ with residue field $F$, and $\pi$ be a prime element of $O_{K}$. The homomorphisms

$$
\begin{gathered}
\Omega_{F}^{1} \longrightarrow \mathcal{U}^{i} K_{2}(K) / U^{i+1} K_{2}(K) \\
x \cdot d y / y \mapsto\left\{1+\pi^{i} \widetilde{x}, \widetilde{y}\right\},
\end{gathered}
$$

and

$$
\begin{aligned}
F \longrightarrow & U^{i} K_{2}(K) / \mathcal{U}^{i} K_{2}(K) \\
x & \left.\mapsto 1+\pi^{i} \widetilde{x}, \pi\right\},
\end{aligned}
$$


( $\widetilde{x}$ and $\widetilde{y}$ are liftings of $x$ and $y$ to $O_{K}$, and the classes of the symbols do not depend on the choices) are surjective. They determined the kernels of the above homomorphisms in the range $0<i \leq e p /(p-1)$ where $e=v_{K}(p)$. In particular, for our $K$, the above homomorphisms (2) and (3) induce isomorphisms

(i) $\Omega_{F}^{1} \stackrel{\simeq}{\longrightarrow} \operatorname{gr}^{i} K_{2}(K)$ for $i=1,2, \ldots, p-1$, and $p+1$.

(ii) $\left\{\begin{array}{l}F / F^{p} \stackrel{\simeq}{\longrightarrow} U^{p} K_{2}(K) / \mathcal{U}^{p} K_{2}(K) \\ \Omega_{F}^{1} / B_{1} \Omega_{F}^{1} \simeq \mathcal{U}^{p} K_{2}(K) / U^{p+1} K_{2}(K) .\end{array}\right.$

We also remark the surjectivity of (2) and (3) implies that $\mathcal{U}^{i} K_{2}(K) / U^{i+1} K_{2}(K)$ is generated by the image of $\left\{U_{K}^{i}, T\right\}$, and that $U^{i} K_{2}(K) / \mathcal{U}^{i} K_{2}(K)$ is generated by the image of $\left\{U_{K}^{i}, \pi\right\}$ in our case.

Let $U^{i}\left(K_{2}(K) / p\right)$ be the filtration on $K_{2}(K) / p$, induced from the filtration $U^{i} K_{2}(K)$. We put $\operatorname{gr}^{i}\left(K_{2}(K) / p\right)=U^{i}\left(K_{2}(K) / p\right) / U^{i+1}\left(K_{2}(K) / p\right)$. Bloch and Kato [2] also determined the structure of $\mathrm{gr}^{i}\left(K_{2}(K) / p\right)$ for general complete discrete valuation field $K$. In our case, $(2)$ and (3) induce isomorphisms

(iii) $\Omega_{F}^{1} \stackrel{\simeq}{\longrightarrow} \operatorname{gr}^{i}\left(K_{2}(K) / p\right)$ for $i=1,2, \ldots, p-1$, and $p+1$, and

(iv) $F / F^{p} \stackrel{\simeq}{\longrightarrow} \operatorname{gr}^{p}\left(K_{2}(K) / p\right) \quad\left(x \mapsto\left\{1+\pi^{p} \widetilde{x}, \pi\right\}\right)$. Here, we have $\mathcal{U}^{p}\left(K_{2}(K) / p\right)=U^{p+1} K_{2}(K)$.

These results will be used in the subsequent sections.

Corollary 1.3. $K$ does not have a cyclic extension which is totally ramified and which is of degree $p^{3}$.

Proof. Let $M / K$ be a totally ramified, cyclic extension of degree $p^{n}$. In order to show $n \leq 2$, since $M / K$ is wildly ramified, it suffices to show that $p^{2}\left(U^{1} K_{2}(K) / U^{1} K_{2}(K) \cap N_{M / K} K_{2}(M)\right)=0$ where $N_{M / K}$ is the norm map. In fact, if $K$ is a 2-dimensional local field in the sense of Kato [8] and Parshin [18], this is clear from the isomorphism theorem of local class field theory

$$
K_{2}(K) / N_{M / K} K_{2}(M) \simeq \operatorname{Gal}(M / K) .
$$

In general case, $U^{1} K_{2}(K) / U^{1} K_{2}(K) \cap N_{M / K} K_{2}(M)$ contains an element of order $p^{n}$ by Lemma (3.3.4) in [12]. So it suffices to show

$$
p^{2}\left(U^{1} K_{2}(K) / U^{1} K_{2}(K) \cap N_{M / K} K_{2}(M)\right)=0 .
$$

We will first prove that $U^{p+2} K_{2}(K) \subset N_{M / K} K_{2}(M)$. If $j$ is sufficiently large, $U_{K}^{j}=1+m_{K}^{j}$ is in $\left(K^{\times}\right)^{p^{n}}$, so $U^{j} K_{2}(K)$ is in $p^{n} K_{2}(K)$, hence in $N_{M / K} K_{2}(M)$. So by Theorem 1.1, in order to prove $U^{p+2} K_{2}(K) \subset$ $N_{M / K} K_{2}(M)$, it suffices to show $\left\{U_{K}^{2 p}, \pi\right\}$ is in $N_{M / K} K_{2}(M)$. Since $M / K$ is totally ramified, there is a prime element $\pi^{\prime}$ of $O_{K}$ such that $\pi^{\prime} \in$ $N_{M / K}\left(M^{\times}\right)$. Hence, the subgroup $\left\{U_{K}^{2 p}, \pi^{\prime}\right\}$ is contained in $N_{M / K} K_{2}(M)$. 
We note that $\left\{U_{K}^{i}, \pi\right\}$ is generated by $\left\{U_{K}^{i}, \pi^{\prime}\right\}$ and $\mathcal{U}^{i} K_{2}(K)$ for all $i>0$. Hence, Theorem 1.1 also tells us that $\left\{U_{K}^{2 p}, \pi\right\}$ is generated by $\left\{U_{K}^{2 p}, \pi^{\prime}\right\}$ and $U^{j} K_{2}(K)$ for sufficiently large $j$. This shows that $\left\{U_{K}^{2 p}, \pi\right\}$ is in $N_{M / K} K_{2}(M)$, and $U^{p+2} K_{2}(K) \subset N_{M / K} K_{2}(M)$.

Since $\left(U_{K}^{1}\right)^{p^{2}} \subset U_{K}^{p+2}$, we get $p^{2} U^{1} K_{2}(K) \subset U^{p+2} K_{2}(K)$, and $p^{2}\left(U^{1} K_{2}(K) / U^{1} K_{2}(K) \cap N_{M / K} K_{2}(M)\right)=0$. This completes the proof of Corollary 1.3.

In order to describe the structure of $K_{2}(K)$, we need the following exponential homomorphism introduced in [12] Lemma 2.4 (see also Lemma 2.2 in $\S 2$ ). We define $K_{2}(K)^{\wedge}$ ( resp. $\hat{\Omega}_{O_{K}}^{1}$ ) to be the $p$-adic completion of $K_{2}(K)$ (resp. $\Omega_{O_{K}}^{1}$ ). Then, there is a homomorphism

$$
\exp _{p^{2}}: \hat{\Omega}_{O_{K}}^{1} \longrightarrow K_{2}(K)^{\wedge}
$$

such that $a \cdot d b \mapsto\left\{\exp \left(p^{2} a b\right), b\right\}$ for $a \in O_{K}$ and $b \in O_{K} \backslash\{0\}$. Here $\exp (x)=\Sigma_{n \geq 0} x^{n} / n$ !. Concerning $K_{2}(K)^{\wedge}$, we have

Corollary 1.4. Let $K$ be as in Theorem 1.1. Then, the image of

$$
\exp _{p^{2}}: \hat{\Omega}_{O_{K}}^{1} \longrightarrow K_{2}(K)^{\wedge}
$$

is $\mathcal{U}^{2 p} K_{2}(K)^{\wedge}$ and the kernel is the $\mathbf{Z}_{p}$-module generated by da with $a \in O_{K}$ and $b(p d \pi / \pi-d T / T)$ with $b \in O_{K}$.

We will prove this corollary in the end of $\S 3$.

\section{2. $p$-torsions of $K_{2}(K)$}

Let $\zeta$ be a primitive $p$-th root of unity. We define $L_{0}=K_{0}(\zeta)$ and $L=K(\zeta)=L_{0}(\pi)$ where $\pi^{p}=p T$ as in Theorem 1.1.

Let $\left\{U^{i} K_{2}(L)\right\}$ be the filtration on $K_{2}(L)$ defined similarly $\left(U^{i} K_{2}(L)\right.$ is a subgroup generated by $\left\{1+m_{L}^{i}, L^{\times}\right\}$where $m_{L}$ is the maximal ideal of $\left.O_{L}\right)$. Since $L / K$ is a totally ramified extension of degree $p-1$, we have natural maps $U^{i} K_{2}(K) \longrightarrow U^{(p-1) i} K_{2}(L)$.

We also use the filtration $U^{i}\left(K_{2}(L) / p\right)$ on $K_{2}(L) / p$, induced from the filtration $U^{i} K_{2}(L)$. If $\eta$ is in $U^{i}\left(K_{2}(L) / p\right) \backslash U^{i+1}\left(K_{2}(L) / p\right)$, we write $\mathrm{fil}_{L}(\eta)=i$. We also note that since $L / K$ is of degree $p-1, U^{i}\left(K_{2}(K) / p\right) \longrightarrow$ $U^{(p-1) i}\left(K_{2}(L) / p\right)$ is injective.

Our aim in this section is to prove the following Lemma 2.1.

Lemma 2.1. Suppose $a \in U_{K_{0}}=O_{K_{0}}^{\times}$.

(1) We have $\left\{\zeta, 1+\left(\pi^{i} /(\zeta-1)\right) a\right\} \equiv\left\{1-\pi^{i} a, T\right\}\left(\bmod U^{(p-1) i+1} K_{2}(L)\right)$ for $i>1$. 
(2) We regard $\left\{\zeta, 1+\left(\pi^{i} /(\zeta-1)\right) a\right\}$ as an element of $K_{2}(L) / p$. For $i=$ $2, \ldots, p-1$ and $p+1$, we have

$$
\operatorname{fil}_{L}\left(\left\{\zeta, 1+\left(\pi^{i} /(\zeta-1)\right) a\right\} \bmod p\right)=(p-1) i .
$$

If $i=p$, then $\operatorname{fil}_{L}\left(\left\{\zeta, 1+\left(\pi^{p} /(\zeta-1)\right) a\right\} \bmod p\right)>(p-1) p$.

(3) For $i=p+2$,

$$
\left\{\zeta, 1+\left(\pi^{p+2} /(\zeta-1)\right) a\right\}=\left\{\exp \left(\pi^{p+2} a\right), p\right\}
$$

in $K_{2}(L)^{\wedge}$ where $K_{2}(L)^{\wedge}$ is the $p$-adic completion of $K_{2}(L)$ and $\exp (x)=$ $\Sigma_{n \geq 0} x^{n} / n !$.

(4) For $i>p+2,\left\{\zeta, 1+\left(\pi^{i} /(\zeta-1)\right) a\right\}=0 \quad$ in $K_{2}(L)^{\wedge}$.

We introduced the map $\exp _{p^{2}}$ in Corollary 1.4 , but more generally, we can define $\exp _{p}$ as in the following lemma, whose proof will be done in Appendix Corollary A2.10 (see also Remark A2.11). The existence of $\exp _{p^{2}}$ follows at once from the existence of $\exp _{p}$. For more general exponential homomorphism $\left(\exp _{c}\right.$ with smaller $\left.v_{K}(c)\right)$, see [15].

Lemma 2.2. Let $K$ be a complete discrete valuation field of mixed characteristics $(0, p)$. As in $\S 1$, we denote by $K_{2}(K)^{\wedge}\left(\right.$ resp. $\left.\hat{\Omega}_{O_{K}}^{1}\right)$ the p-adic completion of $K_{2}(K)$ (resp. $\Omega_{O_{K}}^{1}$ ). Then there exists a homomorphism

$$
\exp _{p}: \hat{\Omega}_{O_{K}}^{1} \longrightarrow K_{2}(K)^{\wedge}
$$

such that $a \cdot d b \mapsto\{\exp (p a b), b\}$ for $a \in O_{K}$ and $b \in O_{K} \backslash\{0\}$.

We use the following consequence of Lemma 2.2.

Corollary 2.3. In the notation of Lemma 2.2, we have

$$
\left\{1+p^{2} c, p\right\}=0 \quad \text { in } \quad K_{2}(K)^{\wedge}
$$

for any $c \in O_{K}$.

Proof. In fact, $\left\{1+p^{2} c, p\right\}=\exp _{p}\left(p^{-2} \log \left(1+p^{2} c\right) \cdot d p\right)$. Hence, by Lemma 2.2 and $d p=0$, we get the conclusion.

We also use the following lemma in Kato [7].

Lemma 2.4. (Lemma 6 in [7]) If $x \neq 0,1$, and $y \neq 1, x^{-1}$,

$$
\{1-x, 1-y\}=\{1-x y,-x\}+\{1-x y, 1-y\}-\{1-x y, 1-x\}
$$

Proof of Lemma 2.4 .

$$
\begin{aligned}
\{1-x, 1-y\} & =\{1-x, x(1-y)\}=\{1-x,-((1-x)-(1-x y))\} \\
& =\left\{1-x, 1-\frac{1-x y}{1-x}\right\}=\left\{1-x y, 1-\frac{1-x y}{1-x}\right\} \\
& =\left\{1-x y,-x(1-y)(1-x)^{-1}\right\}
\end{aligned}
$$


Using this lemma, we have

$$
\begin{aligned}
\{\zeta, 1+ & \left.\left(\pi^{i} /(\zeta-1)\right) a\right\}= \\
& \left\{1-\pi^{i} a, \zeta-1\right\}+\left\{1-\pi^{i} a, 1+\left(\pi^{i} /(\zeta-1)\right) a\right\}+\left\{\zeta, 1-\pi^{i} a\right\}
\end{aligned}
$$

Put $\pi_{L}=(\zeta-1) / \pi$, and $u=p /(\zeta-1)^{p-1}$. Then $\pi_{L}$ is a prime element of $O_{L}$, and $u$ is a unit of $\mathbf{Z}_{p}[\zeta]$. Since $\pi^{p}=p T$, we have

$$
\zeta-1=u \pi_{L}^{p} T
$$

Since $u=v^{p}(1+w(\zeta-1))$ for some $v, w$ in $\mathbf{Z}_{p}[\zeta]$, by (4) and (5) we get

$$
\left\{\zeta, 1+\left(\pi^{i} /(\zeta-1)\right) a\right\} \equiv\left\{1-\pi^{i} a, T\right\} \quad\left(\bmod U^{(p-1) i+1} K_{2}(L)\right) .
$$

Thus, we obtain Lemma 2.1 (1).

Put $x=a \bmod p \in F$. Lemma 2.1 (2) follows from Lemma 2.1 (1). In fact, if $1<i<p$ or $i=p+1,\left\{1-\pi^{i} a, T\right\} \bmod p$ is not in $U^{i+1}\left(K_{2}(K) / p\right)$ by Remark 1.2 (iii) (note that $x \neq 0$ and $x \cdot d t / t \neq 0$ ). Hence, it is not in $U^{(p-1) i+1}\left(K_{2}(L) / p\right)$. So, $\operatorname{fil}_{L}\left(\left\{1-\pi^{i} a, T\right\} \bmod p\right)=(p-1) i$. For $i=p,\left\{1-\pi^{p} a, T\right\} \bmod p$ is in $U^{p+1}\left(K_{2}(K) / p\right)$. In fact, we may suppose $a=\Sigma_{i=0}^{p-1} b_{i}^{p} T^{i}$ for some $b_{i} \in O_{K}$, then $\left\{1-\pi^{p} a, T\right\} \equiv \Sigma_{i \geq 1}\left\{1-b_{i}^{p} T^{i}, T\right\}=$ $-\Sigma_{i \geq 1} i^{-1}\left\{1-b_{i}^{p} T^{i}, b_{i}^{p}\right\} \equiv 0\left(\bmod U^{p+1}\left(K_{2}(K) / p\right)\right)(\mathrm{cf}$. Remark $1.2(\mathrm{iv}))$. Hence, $\operatorname{fil}_{L}\left(\left\{1-\pi^{p} a, T\right\} \bmod p\right)>(p-1) p$.

If $p>3$ or $i>p+3$, Lemma 2.1 (4) is easy because $1+\left(\pi^{i} /(\zeta-1)\right) a$ is in $U_{L}^{p^{2}+1} \subset\left(L^{\times}\right)^{p}$ (Note that $\left.e_{L} p /(p-1)=p^{2}\right)$. We deal with the case $p=3$ and $i=p+3$ in the end of this section.

We proceed to the proof of Lemma $2.1(3)$. Since $\left(1+\left(\pi^{p+2} /(\zeta-\right.\right.$ 1))a) $/\left(1+\left(\pi^{p+2} /(\zeta-1)\right) a \zeta\right) \in U_{L}^{p^{2}+1} \subset\left(L^{\times}\right)^{p}$,

$$
\left\{\zeta, 1+\left(\pi^{p+2} /(\zeta-1)\right) a\right\}=\left\{\zeta, 1+\left(\pi^{p+2} /(\zeta-1)\right) a \zeta\right\}
$$

By Lemma 2.2, we have

$$
\begin{aligned}
\left\{\zeta, 1+\left(\pi^{p+2} /(\zeta-1)\right) a \zeta\right\} & =\left\{\zeta, \exp \left(\pi^{p+2} a \zeta /(\zeta-1)\right)\right\} \\
& =-\exp _{p}\left(\left(\pi^{2} T a /(\zeta-1)\right) d \zeta\right) \\
& =-\exp _{p}\left(\left(\pi^{2} T a /(\zeta-1)\right) d(\zeta-1)\right) \\
& =-\left\{\exp \left(\pi^{p+2} a\right), \zeta-1\right\}
\end{aligned}
$$

Hence by (5) (6) and (7), we obtain

$$
\begin{aligned}
\left\{\zeta, 1+\left(\pi^{p+2} /(\zeta-1)\right) a\right\} & =-\left\{\exp \left(\pi^{p+2} a\right), u\right\} \\
& -p\left\{\exp \left(\pi^{p+2} a\right), \pi_{L}\right\} \\
& -\left\{\exp \left(\pi^{p+2} a\right), T\right\}
\end{aligned}
$$


First of all, $\left\{\exp \left(\pi^{p+2} a\right), u\right\}=0$ in $K_{2}(L)^{\wedge}$. In fact, if we write $d u=w \cdot d \zeta$ for some $w \in \mathbf{Z}_{p}[\zeta]$,

$$
\begin{aligned}
\left\{\exp \left(\pi^{p+2} a\right), u\right\} & =\exp _{p}\left(\pi^{2} T a \cdot d u / u\right) \\
& =\exp _{p}\left(\pi^{2} T a u^{-1} w \cdot d \zeta\right) \\
& =\left\{\exp \left(\pi^{p+2} a w \zeta u^{-1}\right), \zeta\right\} .
\end{aligned}
$$

Since $\exp \left(\pi^{p+2} a w \zeta u^{-1}\right)$ is in $U_{L}^{p^{2}+1} \subset\left(L^{\times}\right)^{p},\left\{\exp \left(\pi^{p+2} a\right), u\right\}=0$.

By the same method, $p\left\{\exp \left(\pi^{p+2} a\right), \zeta-1\right\}=0$, hence the second term of the right hand side of $(8)$ is equal to $p\left\{\exp \left(\pi^{p+2} a\right), \pi\right\}$ (from $\pi_{L}=(\zeta-1) / \pi$ ). Hence by (8) we have

$$
\left\{\zeta, 1+\left(\pi^{p+2} /(\zeta-1)\right) a\right\}=\left\{\exp \left(\pi^{p+2} a\right), \pi^{p} / T\right\}=\left\{\exp \left(\pi^{p+2} a\right), p\right\}
$$

(recall that $\pi^{p}=p T$ ). Thus, we have got Lemma 2.1 (3).

We go back to Lemma 2.1 (4). For $p=3$ and $i=p+3$, by the same method, we obtain

$$
\left\{\zeta, 1+\left(\pi^{p+3} /(\zeta-1)\right) a\right\}=\left\{\exp \left(\pi^{p+3} a\right), p\right\} .
$$

But the right hand side is zero by Corollary 2.3 .

\section{Proof of the theorem}

3.1. First of all, we prove Theorem 1.1 (1). Let $i$ be an integer such that $p+1<i$. Then by Lemma $2.1(1)$, we have

$$
\left\{\zeta, 1+\left(\pi^{i-p} /(\zeta-1)\right) a T\right\} \equiv\left\{1-\pi^{i-p} a T, T\right\} \quad\left(\bmod U^{(p-1)(i-p)+1} K_{2}(L)\right)
$$

for $a \in O_{K_{0}}$. Hence, taking the multiplication by $p$, we get

$$
0 \equiv\left\{1-p \pi^{i-p} a T, T\right\}=\left\{1-\pi^{i} a, T\right\} \quad\left(\bmod U^{(p-1) i+1} K_{2}(L)\right) .
$$

This implies $\left\{1-\pi^{i} a, T\right\} \equiv 0\left(\bmod U^{i+1} K_{2}(K)\right)$. Since $U^{i} K_{2}(K)=$ $\mathcal{U}^{i} K_{2}(K)$ for all $i$ with $(i, p)=1$ and the surjectivity of (2) implies that $\mathcal{U}^{i} K_{2}(K) / U^{i+1} K_{2}(K)$ is generated by the image of $\left\{U_{K}^{i}, T\right\}$, it follows from $\left\{1-\pi^{i} a, T\right\} \in U^{i+1} K_{2}(K)$ that $U^{i} K_{2}(K)=U^{i+1} K_{2}(K)$ for all $i$ with $(i, p)=1$.

We remark that by [12] Theorem 2.2, if $i>2 p$ and $i$ is prime to $p$, we already knew $\operatorname{gr}^{i} K_{2}(K)=0\left(\left(\hat{\Omega}_{O_{K}}^{1}\right)_{\text {tors }}\right.$ is generated by $\pi^{p-1} d \pi-d T$, and isomorphic to $\left.O_{K} /(p)\right)$. So the problem was only to show $\mathrm{gr}^{i} K_{2}(K)=0$ for $i$ such that $p+1<i<2 p$.

3.2. Next we proceed to $i=2 p$. By $\pi^{p}=p T$, we have $p \cdot d T=p \pi^{p} \cdot d \pi / \pi$. Hence, $\exp _{p}(p \cdot d T)=\exp _{p}\left(p \pi^{p} \cdot d \pi / \pi\right)$, namely

$$
\left\{\exp \left(p^{2} a T\right), T\right\}=\left\{\exp \left(p^{2} \pi^{p} a\right), \pi\right\} \text { in } K_{2}(K)^{\wedge}
$$


for all $a \in O_{K}$. Hence, $\mathcal{U}^{2 p} K_{2}(K) \subset U^{2 p+1} K_{2}(K)$ and

$$
\operatorname{gr}^{2 p} K_{2}(K)=U^{2 p} K_{2}(K) / \mathcal{U}^{2 p} K_{2}(K)
$$

(this also follows from [12] Theorem 2.2).

For $a \in U_{K_{0}}$, by an elementary calculation

$$
\begin{aligned}
\left\{1-p \pi^{p} a^{p}, \pi\right\} & \equiv p\left\{1-\pi^{p} a^{p}, \pi\right\} \quad\left(\bmod U^{2 p+1} K_{2}(K)\right) \\
& =\left\{1-\pi^{p} a^{p}, \pi^{p}\right\}=\left\{1-\pi^{p} a^{p}, 1 / a^{p}\right\} \\
& \equiv-\left\{1-p \pi^{p} a^{p}, a\right\},
\end{aligned}
$$

we know that $F^{p}$ is contained in the kernel of the map $x \mapsto\left\{1+p \pi^{p} \widetilde{x}, \pi\right\}$ in (3) from $F$ to $\operatorname{gr}^{2 p} K_{2}(K)$ because of $\mathcal{U}^{2 p} K_{2}(K)=U^{2 p+1} K_{2}(K)$.

Next we assume that $a \in U_{K_{0}}$ and $x=a \bmod p$ is not in $F^{p}$. We will prove $\left\{1+p \pi^{p} a, \pi\right\} \notin U^{2 p+1} K_{2}(K)$. Let $L=K(\zeta), U^{i}\left(K_{2}(L) / p\right), \mathrm{fil}_{L}(\eta)$ be as in $\S 2$. Since $x \notin F^{p}$, by Remark 1.2 (iv) we have $\operatorname{fil}_{K}\left(\left\{1+\pi^{p} a, \pi\right\} \bmod p\right)=p$ and

$$
\operatorname{fil}_{L}\left(\left\{1+\pi^{p} a, \pi\right\} \bmod p\right)=(p-1) p .
$$

Let $\Delta=\operatorname{Gal}(L / K)$ be the Galois group of $L / K$. Consider the following commutative diagram of exact sequences

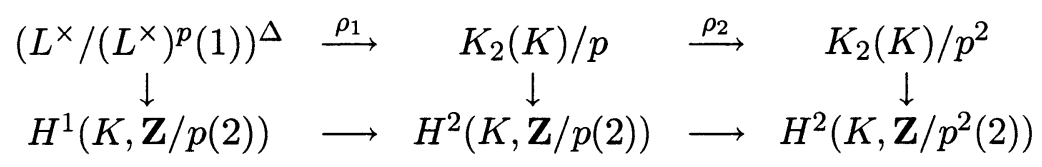

where $\rho_{1}$ is the restriction to the $\Delta$-invariant part $\left(L^{\times} /\left(L^{\times}\right)^{p}(1)\right)^{\Delta}$ of the $\operatorname{map} L^{\times} /\left(L^{\times}\right)^{p}(1) \longrightarrow K_{2}(L) / p ; x \mapsto\{\zeta, x\}$ (we used $\left(K_{2}(L) / p\right)^{\Delta} \simeq$ $\left.K_{2}(K) / p\right)$, and $\rho_{2}$ is the map induced from the multiplication by $p$ $\left(\alpha \bmod p \mapsto p \alpha \bmod p^{2}\right)$. The left vertical arrow is bijective, and the central and the right vertical arrows are also bijective by Mercurjev and Suslin.

This diagram says that the kernel of $\rho_{2}$ is equal to the image of $\left\{\zeta, L^{\times}\right\}^{\Delta}$ in $K_{2}(K) / p$. The filtration $U_{L}^{i}=1+m_{L}^{i}$ on $L^{\times}$induces a filtration on $\left(L^{\times} /\left(L^{\times}\right)^{p}(1)\right)^{\Delta}$, and its graded quotients are calculated as $\left(U_{L}^{i} / U_{L}^{i+1}(1)\right)^{\Delta}$ $=U_{L}^{i} / U_{L}^{i+1}$ if $i \equiv-1 \bmod p-1$, and $=0$ otherwise $\left(\left(L^{\times} / U_{L} \otimes \mathbf{Z} / p(1)\right)^{\Delta}\right.$ also vanishes). Since the image of $1+\left(\pi^{i} /(\zeta-1)\right) U_{K_{0}}$ generates $U_{L}^{(p-1) i-p} / U_{L}^{(p-1) i-p+1}$, if $\eta$ is in Image $\rho_{1} \subset\left(K_{2}(L) / p\right)^{\Delta}$, then $\eta$ can be written as $\eta \equiv\left\{\zeta, 1+\left(\pi^{i} /(\zeta-1)\right) a_{i}\right\}\left(\bmod U^{(p-1) i+1} K_{2}(L)\right)$ for some $i>0$ with $a_{i} \in U_{K_{0}}$. Hence, by Lemma $2.1(2)$, we have $\operatorname{fil}_{L}(\eta) \neq(p-1) p$.

Therefore by (9), $\left\{1+\pi^{p} a, \pi\right\}$ does not belong to $\left\{\zeta, L^{\times}\right\}^{\Delta}$ in $\left(K_{2}(L) / p\right)^{\Delta}=K_{2}(K) / p$. So by the above exact sequence,

$$
\left\{1+p \pi^{p} a, \pi\right\} \neq 0 \quad \text { in } \quad K_{2}(K) / p^{2} .
$$

Since $U^{2 p+1} K_{2}(K)=U^{3 p} K_{2}(K) \subset p^{2} K_{2}(K)$, this implies that $\left\{1+p \pi^{p} a, \pi\right\}$ $\neq 0$ in $\operatorname{gr}^{2 p} K_{2}(K)$. Hence, the kernel of the map $x \mapsto\left\{1+p \pi^{p} \widetilde{x}, \pi\right\}$ from 
$F$ to $\operatorname{gr}^{i} K_{2}(K)$, coincides with $F^{p}$. This completes the proof of Theorem $1.1(2)$.

3.3. We next prove (3) of Theorem 1.1. Let $n \geq 3$. By the same method as in 3.2 , we have $\mathcal{U}^{n p} K_{2}(K)=U^{n p+1} K_{2}(K)$ (this also follows from [12] Theorem 2.2), in particular, the map

$$
F \longrightarrow \operatorname{gr}^{n p} K_{2}(K) \quad\left(x \mapsto\left\{1+p^{n} \widetilde{x}, \pi\right\}\right)
$$

is surjective.

Suppose that $a \in O_{K_{0}}$. By Corollary 2.3, we have $\left\{\exp \left(p^{n-1} a\right), p\right\}=0$ in $K_{2}(K)^{\wedge}$, hence we get

$$
\begin{aligned}
\left\{\exp \left(p^{n} a\right), \pi\right\} & =\left\{\exp \left(p^{n-1} a\right), \pi^{p}\right\}=\left\{\exp \left(p^{n-1} a\right), p T\right\} \\
& =\left\{\exp \left(p^{n-1} a\right), T\right\} .
\end{aligned}
$$

Since $n \geq 3$, the above formula implies

$$
\begin{aligned}
\left\{1+p^{n} a, \pi\right\} & \equiv\left\{\exp \left(p^{n} a\right), \pi\right\} \quad\left(\bmod U^{(n+1) p} K_{2}(K)\right) \\
& =\left\{\exp \left(p^{n-1} a\right), T\right\} \\
& \equiv\left\{1+p^{n-1} a, T\right\} \quad\left(\bmod U^{(n+1) p} K_{2}(K)\right) .
\end{aligned}
$$

Recall that we fixed a $p$-base $t$ of $F$ such that $T \bmod p=t$. We define subgroups $\mathcal{B}_{n}$ of $F$ by $\mathcal{B}_{n} d t / t=B_{n} \Omega_{F}^{1}$ for $n>0$. Suppose that $x$ is in $\mathcal{B}_{n-2}$. Let $a=\widetilde{x}$ be a lifting of $x$ to $O_{K_{0}}$. Then by [14] Proposition 2.3, we get

$$
\left\{1+p^{n-1} a, T\right\} \in U^{n} K_{2}\left(K_{0}\right) .
$$

Let $i_{K / K_{0}}: K_{2}\left(K_{0}\right) \longrightarrow K_{2}(K)$ be the natural map. Then, we have $i_{K / K_{0}}\left(U^{n} K_{2}\left(K_{0}\right)\right) \subset U^{n p} K_{2}(K)$, but by the formula (11), $i_{K / K_{0}}\left(U^{n-1} K_{2}\left(K_{0}\right)\right) \subset U^{n p} K_{2}(K)$ also holds. Hence by (12), (13), and $i_{K / K_{0}}\left(U^{n} K_{2}\left(K_{0}\right)\right) \subset U^{(n+1) p} K_{2}(K)$, we know that $\left\{1+p^{n} a, \pi\right\}$ is in $U^{(n+1) p} K_{2}(K)$. Namely, $\mathcal{B}_{n-2}$ is in the kernel of the map (10).

Since $B_{n-2} \Omega_{F}^{1}$ is generated by the elements of the form $x^{p^{n-2}} t^{i} \cdot d t / t$ such that $x \in F$ and $1 \leq i \leq p^{n-2}-1, F / \mathcal{B}_{n-2}$ is isomorphic to $F^{p^{n-2}}$, and we obtain a surjective homomorphism

$$
F^{p^{n-2}} \longrightarrow \operatorname{gr}^{n p} K_{2}(K) ; \quad x \mapsto\left\{1+p^{n} \widetilde{x}, \pi\right\} .
$$

We proceed to the proof of the injectivity of (14). We assume that $\left\{1+p^{n} a, \pi\right\}$ is in $U^{n p+1} K_{2}(K)$ for $a \in O_{K_{0}}$. Since $U^{n p+1} K_{2}(K)=$ $U^{(n+1) p} K_{2}(K) \subset p^{n} K_{2}(K),\left\{1+p^{n} a, \pi\right\}=0$ in $K_{2}(K) / p^{n}$. Hence $\left\{1+p^{n-1} a, \pi\right\}$ is in the kernel of $K_{2}(K) / p^{n-1} \longrightarrow K_{2}(K) / p^{n}\left(\alpha \bmod p^{n-1} \mapsto\right.$ 
$\left.p \alpha \bmod p^{n}\right)$. As in 3.2 , we consider a commutative diagram of exact sequences with vertical bijective arrows

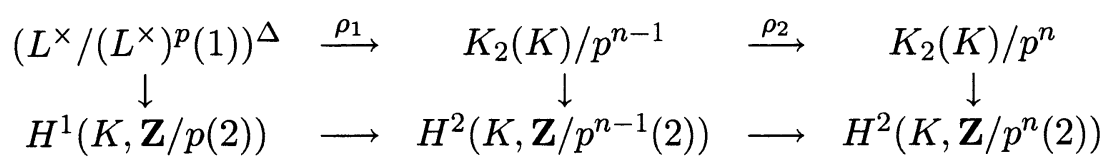

where $\rho_{1}$ is the restriction to $\left(L^{\times} /\left(L^{\times}\right)^{p}(1)\right)^{\Delta}$ of the map $L^{\times} /\left(L^{\times}\right)^{p}(1) \longrightarrow$ $K_{2}(L) / p^{n-1} ; x \mapsto\{\zeta, x\}$ (we also used $\left(K_{2}(L) / p^{n-1}\right)^{\Delta} \simeq K_{2}(K) / p^{n-1}$ ). From this diagram, we know that $\left\{1+p^{n-1} a, \pi\right\}$ is in the image of $\rho_{1}$. We write $\left\{1+p^{n-1} a, \pi\right\}=\{\zeta, c\}$ for some $c \in\left(L^{\times} /\left(L^{\times}\right)^{p}(1)\right)^{\Delta}$. So by the argument in 3.2, $c$ is in $U_{L}^{(p-1) i-p}$ for some $i>1$. If $c$ was in $U_{L}^{(p-1) i-p} \backslash$ $U_{L}^{(p-1)(i+1)-p}$ for some $i$ with $1<i<p$, we would have by Lemma 2.1 (2) $\mathrm{fil}_{L}(\{\zeta, c\} \bmod p)=(p-1) i$. But $\left\{1+p^{n-1} a, \pi\right\}$ is zero in $K_{2}(L) / p$ (because $\left.1+p^{n-1} a \in\left(L^{\times}\right)^{p}\right)$, so $c$ must be in $U_{L}^{(p-1) p-p}(1)^{\Delta}$. We write $c=c_{1} c_{2}$ with $c_{1} \in U_{L_{0}}^{p-2}(1)^{\Delta}$ and $c_{2} \in U_{L}^{(p-1)(p+1)-p}(1)^{\Delta}$. Again by the same argument using Lemma $2.1(2), c_{2}$ must be in $U_{L}^{(p-1)(p+2)-p}(1)^{\Delta}$. By Lemma 2.1 (3) and (4), we can write

$$
\left\{1+p^{n-1} a, \pi\right\}=\left\{\zeta, c_{1}\right\}+\left\{\exp \left(\pi^{p+2} c_{3}\right), p\right\}
$$

for some $c_{3} \in O_{K_{0}}$ in $K_{2}(L) / p^{n-1}$. Let $N_{L / L_{0}}: K_{2}(L) \longrightarrow K_{2}\left(L_{0}\right)$ be the norm homomorphism. Taking the norm $N_{L / L_{0}}$ of the both sides of the equation (15), we get

$$
\begin{aligned}
\left\{1+p^{n-1} a, p T\right\} & =\left\{\zeta, c_{1}^{p}\right\}+\left\{\exp \left(\operatorname{Tr}_{L / L_{0}}\left(\pi^{p+2} c_{3}\right)\right), p\right\} \\
& =0
\end{aligned}
$$

where $\operatorname{Tr}_{L / L_{0}}$ is the trace, and we used $\operatorname{Tr}_{L / L_{0}}\left(\pi^{p+2} c_{3}\right)=p T c_{3} \operatorname{Tr}_{L / L_{0}}\left(\pi^{2}\right)=$ 0 . On the other hand, the left hand side of (16) is equal to $\left\{1+p^{n-1} a, T\right\}$ by Corollary 2.3. Hence, the equation (16) implies that $\left\{1+p^{n-1} a, T\right\}=0$ in $K_{2}\left(L_{0}\right) / p^{n-1}$, hence in $K_{2}\left(K_{0}\right) / p^{n-1}$.

In the proof of [14] Corollary 2.5, we showed that $\exp _{p^{2}}$ induces

$$
\exp _{p^{2}}:\left(\Omega_{O_{K_{0}}}^{1} / d O_{K_{0}}\right) \otimes \mathbf{Z} / p^{n-2} \longrightarrow K_{2}\left(K_{0}\right) / p^{n-1}
$$

which is injective. In $K_{2}\left(K_{0}\right) / p^{n-1}$, we have $\left\{\exp \left(p^{n-1} a\right), T\right\}=\{1+$ $\left.p^{n-1} a, T\right\}=0$, hence by the injectvity of the above map, we know that $p^{n-3} a d T / T \bmod p^{n-2}$ is in $d\left(O_{K_{0}} / p^{n-2}\right)$. This implies that $x \cdot d t / t$ is in $B_{n-2} \Omega_{F}^{1}$ where $x=a \bmod p \in F$ ([6] Corollaire 2.3.14 in Chapter 0). Hence, $x$ is in $\mathcal{B}_{n-2}$. Thus, the kernel of the map (10) coincides with $\mathcal{B}_{n-2}$. Namely, the map (14) is bijective. This completes the proof of Theorem 1.1. 


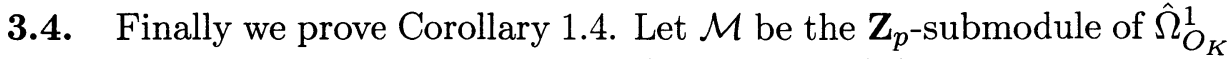
generated by $d a$ with $a \in O_{K}$ and $b(p d \pi / \pi-d T / T)$ with $b \in O_{K}$. It follows from $\pi^{p}=p T$ that $p \pi^{p} d \pi / \pi=p^{2} T d \pi / \pi=p d T$ in $\hat{\Omega}_{O_{K}}^{1}$. Hence, the existence of $\exp _{p}$ implies that $b(p d \pi / \pi-d T / T)$ is in the kernel of $\exp _{p^{2}}$. Further, $d a$ is also in the kernel of $\exp _{p^{2}}$ by Lemma A2.3 in Appendix. So $\exp _{p^{2}}$ factors through $\hat{\Omega}_{O_{K}}^{1} / \mathcal{M}$. Since $b(p d \pi / \pi-d T / T) \in \mathcal{M}, \hat{\Omega}_{O_{K}}^{1} / \mathcal{M}$ is generated by the classes of the form $c d \pi / \pi$. We define $\mathrm{Fil}^{i}$ to be the

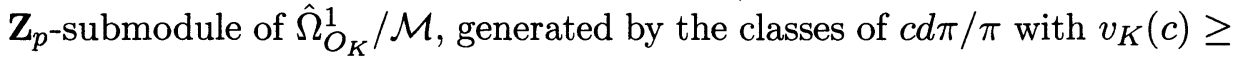
$i-2 p$, and consider $\mathrm{gr}^{i}=\mathrm{Fil}^{i} / \mathrm{Fil}^{i+1}$.

We can easily see that $\operatorname{gr}^{i}=0$ for $i$ which is prime to $p$. In fact, if $i$ is prime to $p$, for $a \in U_{K}, a \pi^{i} d \pi / \pi=a i^{-1} d \pi^{i} \equiv-\pi^{i} d a(\bmod \mathcal{M})$. We can write $d a=a_{1} d T+a_{2} d \pi \equiv a_{1} T p d \pi / \pi+a_{2} \pi d \pi / \pi(\bmod \mathcal{M})$ for some $a_{1}$, $a_{2} \in O_{K}$, hence $a \pi^{i} d \pi / \pi$ is in $\mathrm{Fil}^{i+1}$. For $i \leq 2 p$, we also have $\mathrm{gr}^{i}=0$.

Suppose that $n \geq 3$ and consider a homomorphism

$$
F \longrightarrow \mathrm{gr}^{n p} ; \quad x \mapsto p^{n-2} \widetilde{x} d \pi / \pi .
$$

This does not depend on the choice of $\widetilde{x}$. Suppose that $x$ is in $\mathcal{B}_{n-2}$ (for $\mathcal{B}_{n-2}$, see the previous subsection). We write $x=\Sigma_{i=1}^{p^{n-2}-1} x_{i}^{p^{n-2}} t^{i} \cdot d t / t$ as in 3.3, and take a lifting $a=\Sigma_{i=1}^{p^{n-2}-1} \widetilde{x}_{i}^{p^{n-2}} T^{i} \cdot d T / T$ where $\widetilde{x}_{i}$ is a lifting of $x_{i}$ to $O_{K_{0}}$. We have $p^{n-2} a d \pi / \pi \equiv p^{n-3} a d T / T(\bmod \mathcal{M}) \equiv d b\left(\bmod \mathrm{Fil}^{n p}\right)$ for some $b \in O_{K_{0}}$. Hence, $p^{n-2} a d \pi / \pi \in \mathcal{M}$, and $\mathcal{B}_{n-2}$ is in the kernel of (17). So the restriction of (17) to $F^{p^{n-2}}$ gives a surjective homomorphism $F^{p^{n-2}} \longrightarrow \mathrm{gr}^{n p}$ as in 3.3. This is also injective because the composite $F^{p^{n-2}} \longrightarrow \mathrm{gr}^{n p} \longrightarrow \mathrm{gr}^{n p} K_{2}(K)$ with the induced map by $\exp _{p^{2}}$ is bijective by Theorem 1.1 (3). Therefore, comparing $\mathrm{gr}^{i}$ with $\mathrm{gr}^{i} K_{2}(K)$, we know that $\exp _{p^{2}}: \hat{\Omega}_{O_{K}}^{1} / \mathcal{M} \longrightarrow \mathcal{U}^{2 p} K_{2}(K)^{\wedge}$ is bijective.

\section{Appendix A. Milnor $K$-groups of a local ring over a ring of $p$-adic integers}

In this appendix, we show the existence of $\exp _{p}$ (Corollary A2.10). To do so, we describe the Milnor $K$-groups of a local ring over a complete discrete valuation ring of mixed characteristics, by using the modules of differentials with certain divided power envelopes. (For the precise statement, see Proposition A1.3 and Theorem A2.2.) This description is related to the theory of syntomic cohomology developed by Fontaine and Messing.

On a variety over a complete discrete valuation ring of mixed characteristics, Fontaine and Messing [4] developed the theory of syntomic cohomology which relates the etale cohomology of the generic fiber with the crystalline cohomology of the special fiber. In [9] Kato studied the image of the syntomic cohomology in the derived category of the etale sites, and considered the syntomic complex on the etale site. He also used the Milnor $K$-groups 
in order to relate the syntomic complex with the $p$-adic etale vanishing cycles, and obtain an isomorphism between the sheaf of the Milnor $K$-groups and the cohomology of the syntomic complex after tensoring with an algebraically closed field ([9] Chap.I 4.3, 4.11, 4.12). Our description of the Milnor $K$-groups says that this isomorphism exists without tensoring with an algebraically closed field (for the precise statement cf. Remark A2.12 (28)). This appendix is a part of the author's master's thesis in 1986.

\section{A.1. Smooth case.}

A.1.1. Let $\Lambda$ be a complete discrete valuation ring of mixed characteristics $(0, p)$. We further assume that $p$ is an odd prime number, and that $\Lambda$ is absolutely unramified, namely $p \Lambda$ is the maximal ideal of $\Lambda$. We denote by $F=\Lambda / p \Lambda$ the residue field of $\Lambda$.

Let $\left(R, m_{R}\right)$ be a local ring over $\Lambda$ such that $R / p R$ is essentially smooth over $F$, and $R$ is flat over $\Lambda$. Further, we assume that $R$ is $p$-adically complete, i.e. $R \stackrel{\simeq}{\longrightarrow} \lim R / p^{n} R$, and define $B=R[[X]]$. In this section, we study the Milnor $K$-group of $B$. (One can deal with more general rings by the method in this section, but for simplicity we restrict ourselves to the above ring.)

Since $R / p R$ is essentially smooth over $F, R / p R$ has a $p$-base. Namely, there exists a family $\left(e_{\lambda}\right)_{\lambda \in L}$ of elements of $R / p R$ such that any $a \in R / p R$ can be written uniquely as

$$
a=\sum_{s} a_{s}^{p} \prod_{\lambda \in L} e_{\lambda}^{s(\lambda)}
$$

where $a_{s} \in R / p R$, and $s$ ranges over all functions $L \longrightarrow\{0,1, \ldots, p-1\}$ with finite supports.

For a ring $A, \Omega_{A}^{1}$ denotes the module of Kähler differentials. Let $e_{\lambda}$ be as above, then $\left\{d e_{\lambda}\right\}$ is a basis of the free module $\Omega_{R / p R}^{1}$. We consider a lifting $I \subset R$ of a $p$-base $\left\{e_{\lambda}\right\}$. Then $\{d T ; T \in I\}$ gives a basis of the free $R$-module $\hat{\Omega}_{R}^{1}$ where $\hat{\Omega}_{R}^{1}$ is the $p$-adic completion of $\Omega_{R}^{1}$. Since $R$ is local, we can take $I$ from $R^{\times}$. In the following, we fix $I$ such that $I \subset R^{\times}$.

For the lifting $I$ of a $p$-base, we can take an endomorphism $f$ of $R$ such that $f(T)=T^{p}$ for any $T \in I$, and that $f(x) \equiv x^{p}(\bmod p)$ for any $x \in R$. We fix this endomorphism $f$, and call it the frobenius endomorphism relative to $I$.

We put $B=R[[X]]$. We extend $f$ to an endomorphism of $B$ by $f(X)=$ $X^{p}$. So $f$ satisfies $f(x) \equiv x^{p}(\bmod p)$ for any $x \in B$. Let $X B$ be the ideal of $B$ generated by $X$.

Lemma A.1.1. Put $f_{1}=\frac{1}{p} f: B[1 / p] \longrightarrow B[1 / p], f_{1}^{n}=f_{1} \circ \ldots \circ f_{1}(n$ times $)$, and $E_{1}=\exp \left(\sum_{n=0}^{\infty} f_{1}^{n}\right)$. Then, for $a \in B, E_{1}(a X)$ is in $B^{\times}$, and 
$E_{1}$ defines a homomorphism (Shafarevich function)

$$
E_{1}: X B \longrightarrow B^{\times} \text {. }
$$

It suffices to show $E_{1}(a X)$ is in $B^{\times}$for $a \in B$. We define $a_{n} \in B$ inductively by $a_{0}=a$ and

$$
f^{n}(a)=W_{n}\left(a_{0}, a_{1}, \ldots, a_{n}\right)
$$

where $f^{n}=f \circ \ldots \circ f(n$ times $)$, and $W_{n}\left(T_{0}, \ldots, T_{n}\right)$ is the Witt polynomial ([19] Chap.II). (It is easily verified that $f^{n}(a)-\left(a^{p^{n}}+p a_{1}^{p^{n-1}}+\ldots+p^{n-1} a_{n-1}\right)$ is divisible by $p^{n}$. Hence, $a_{n}$ is well-defined.) By the formula of Artin-Hasse exponential $\exp \left(\Sigma_{n=0}^{\infty} T^{p^{n}} / p^{n}\right)=\Pi_{(p, m)=1}\left(1-T^{m}\right)^{-\mu(m) / m}$, we have

$$
E_{1}(a X)=\prod_{n=0}^{\infty} \prod_{(p, m)=1}\left(1-\left(a_{n} X^{p^{n}}\right)^{m}\right)^{-\mu(m) / m} .
$$

Hence, $E_{1}(a X) \in B^{\times}$.

A.1.2. Let $\hat{\Omega}_{B}^{1}$ be the $(p, X)$-adic completion of $\Omega_{B}^{1}$. For an integer $r \in \mathbf{Z}$, let $\hat{\Omega}_{B}^{r}=\wedge_{B}^{r} \hat{\Omega}_{B}^{1}$ for $r \geq 0$, and $\hat{\Omega}_{B}^{r}=0$ for $r<0$. Let $r$ be positive. Then $f$ naturally acts on $\hat{\Omega}_{B}^{r}$, and the image is contained in $p^{r} \hat{\Omega}_{B}^{r}$. So we can define $f_{r}=p^{-r} f$ on $\hat{\Omega}_{B}^{r}$.

We define $I_{B}=I \cup\{X\}$. Then $\left\{d T_{1} \wedge \ldots \wedge d T_{r} ; T_{i} \in I_{B}\right\}$ is a base of $\hat{\Omega}_{B}^{r}$. For $i>0$ we define $U_{X}^{i} \hat{\Omega}_{B}^{r}$ to be the subgroup (topologically) generated by the elements of the form $a d T_{1} \wedge \ldots \wedge d T_{r}$, and $b d T_{1} \wedge \ldots \wedge d T_{r-1} \wedge d X$ where $a \in X^{i} B, b \in X^{i-1} B$, and $T_{1}, \ldots, T_{r} \in I$.

A.1.3. For a ring $k$ and $q \geq 0$, the Milnor $K$-group $K_{q}^{M}(k)$ is by definition

$$
K_{q}^{M}(k):=\left(k^{\times} \otimes \ldots \otimes k^{\times}\right) / J
$$

where $J$ is the subgroup generated by the elements of the form $a_{1} \otimes \ldots \otimes a_{q}$ such that $a_{i}+a_{j}=0$, or 1 for some $i \neq j$. (The class of $a_{1} \otimes \ldots \otimes a_{q}$ is denoted by $\left\{a_{1}, \ldots, a_{q}\right\}$.)

We will define a homomorphism $E_{q}$ which is regarded as $\exp \left(\Sigma_{n=0}^{\infty} f_{q}^{n}\right)$ from the module of the differential $(q-1)$-forms to the $q$-th Milnor $K$ group. First of all, we remark that in $K_{2}^{M}(B)$, the symbol $\{1+X a, X\}$ makes sense for $a \in B$. Namely, for any $x$ in the maximal ideal $m_{B}$, we define $\{1+x a, x\}$ to be

$$
\{1+x a, x\} ;=\left\{\begin{array}{l}
-\{1+x a,-a\} \quad \text { if } a \notin m_{B} \\
\{-(1+a) /(1-x),(1+a x) /(1-x)\}
\end{array} \quad \text { if } a \in m_{B} .\right.
$$

Then, usual relations like $\{1+x y a, x\}+\{1+x y a, y\}=\{1+x y a, x y\}$, $\{1+x a, x\}+\{1+x b, x\}=\{(1+x a)(1+x b), x\},\{1-x, x\}=0$ hold, and the image of $\{1+x a, x\}$ in $K_{2}^{M}(B[1 / x])$ is $\{1+x a, x\}$ ([22], [11]). Hence, 
the notation $\left\{1+X a, b_{1}, \ldots, b_{q-2}, X\right\}$ also makes sense in $K_{q}^{M}(B)$ where $b_{i} \in B^{\times}$.

We define $K_{q}^{M}(B)^{\wedge}$ to be the $(p, X)$-adic completion of $K_{q}^{M}(B)$, namely the completion with respect to the filtration $\left\{V_{i}\right\}$ where $V_{i}$ is the subgroup generated by $\left\{1+(p, X)^{i}, B^{\times}, \ldots, B^{\times}\right\}$. Let $U_{X}^{i} K_{q}^{M}(B)^{\wedge}$ be the subgroup (topologically) generated by $\left\{1+X^{i} B, B^{\times}, \ldots, B^{\times}\right\}$and $\{1+$ $\left.X^{i} B, B^{\times}, \ldots, B^{\times}, X\right\}$. We define

$$
E_{q}: U_{X}^{1} \hat{\Omega}_{B}^{q-1} \longrightarrow K_{q}^{M}(B)^{\wedge}
$$

by

$$
a X \cdot \frac{d T_{1}}{T_{1}} \wedge \ldots \wedge \frac{d T_{q-1}}{T_{q-1}} \mapsto\left\{E_{1}(a X), T_{1}, \ldots, T_{q-1}\right\}
$$

where $a \in B$ and $T_{i} \in I_{B}$. Since $f_{q}\left(a X \cdot\left(d T_{1}\right) / T_{1} \wedge \ldots \wedge\left(d T_{q-1}\right) / T_{q-1}\right)=$ $f_{1}(a X) \cdot\left(d T_{1}\right) / T_{1} \wedge \ldots \wedge\left(d T_{q-1}\right) / T_{q-1}, E_{q}$ can be regarded as $\exp \left(\Sigma_{n=0}^{\infty} f_{q}^{n}\right)$.

Lemma A.1.2. $E_{q}$ vanishes on $U_{X}^{1} \hat{\Omega}_{B}^{q-1} \cap d \hat{\Omega}_{B}^{q-2}=d\left(U_{X}^{1} \hat{\Omega}_{B}^{q-2}\right)$.

We may assume $q=2$. So we have to prove $E_{2}(d(X a))=0$. By the additivity of the claim, we may assume that $a$ is a product of elements of $I_{B}$, namely $a=\Pi T_{i}$ where $T_{i} \in I_{B}$. In particular, $f(a)=a^{p}$. Using $X a=X \Pi T_{i}$, we have

$$
\begin{aligned}
E_{2}(d(X a)) & =\sum\left\{E_{1}(X a), X T_{i}\right\}=\left\{E_{1}(X a), X a\right\} \\
& =\left\{\prod_{(p, m)=1}\left(1-(X a)^{m}\right)^{-\mu(m) / m}, X a\right\} \\
& =\sum_{(p, m)=1}-\mu(m) / m^{2}\left\{1-(X a)^{m},(X a)^{m}\right\} \\
& =0 .
\end{aligned}
$$

This completes the proof of Lemma 2.2 .

A.1.4. For $i>0$ we define $U_{X}^{i}\left(\hat{\Omega}_{B}^{q-1} / d \hat{\Omega}_{B}^{q-2}\right)$ to be the image of $U_{X}^{i} \hat{\Omega}_{B}^{q-1}$ in $\hat{\Omega}_{B}^{q-1} / d \hat{\Omega}_{B}^{q-2}$.

Proposition A.1.3. $E_{q}$ induces an isomorphism

$$
E_{q}: U_{X}^{1}\left(\hat{\Omega}_{B}^{q-1} / d \hat{\Omega}_{B}^{q-2}\right) \stackrel{\simeq}{\longrightarrow} U_{X}^{1} K_{q}^{M}(B)^{\wedge}
$$

which preserves the filtrations.

Proof. Using Vostokov's pairing [23], Kato defined in [9] a symbol map

$$
h_{q}=\left(s_{f, q}, d \log \right): K_{q}^{M}(B) \longrightarrow\left(\hat{\Omega}_{B}^{q-1} / d \hat{\Omega}_{B}^{q-2}\right) \oplus \hat{\Omega}_{B}^{q}
$$


such that

$$
\begin{aligned}
& s_{f, q}\left(\left\{a_{1}, \ldots, a_{q}\right\}\right) \\
& \quad=\sum_{i=1}^{q}(-1)^{i-1} \frac{1}{p} \log \frac{f\left(a_{i}\right)}{a_{i}^{p}} \frac{d a_{1}}{a_{1}} \wedge \ldots \wedge \frac{d a_{i-1}}{a_{i-1}} \wedge \frac{f}{p}\left(\frac{d a_{i+1}}{a_{i+1}}\right) \wedge \ldots \wedge \frac{f}{p}\left(\frac{d a_{q}}{a_{q}}\right)
\end{aligned}
$$

and $d \log \left\{a_{1}, \ldots, a_{q}\right\}=\left(d a_{1}\right) / a_{1} \wedge \ldots \wedge\left(d a_{q}\right) / a_{q}$.

Concerning the map $s_{f, q}$ we will give two remarks. If $T_{1}, \ldots, T_{q-1}$ are in $I_{B}\left(\right.$ and $\left\{a, T_{1}, \ldots, T_{q-1}\right\}$ is defined), then

$$
s_{f, q}\left(\left\{a, T_{1}, \ldots, T_{q-1}\right\}\right)=\frac{1}{p} \log \frac{f(a)}{a^{p}} \frac{d T_{1}}{T_{1}} \wedge \ldots \wedge \frac{d T_{q-1}}{T_{q-1}} .
$$

Here, we are allowed to take $T_{i}=X$. To see this, since the definition of $s_{f, q}$ is compatible with the product structure of the Milnor $K$-group, we may assume $q=2$ and $T_{1}=X$. Since $\{a, X\}$ is defined, by our convention, $a$ can be written as $a=1+b X$. The image of $\{a, X\}$ under the symbol map $K_{2}(B[1 / X]) \longrightarrow \hat{\Omega}_{B[1 / X]}^{1} / d(B[1 / X])$ is $p^{-1} \log \left(f(1+b X) /(1+b X)^{p}\right) d X / X$ which belongs to the image of $\hat{\Omega}_{B}^{1} / d B \longrightarrow \hat{\Omega}_{B[1 / X]}^{1} / d(B[1 / X])$. This map is injective, so $s_{f, 2}(\{a, X\})=p^{-1} \log \left(f(1+b X) /(1+b X)^{p}\right) d X / X$ (cf.also [10] 3.5).

Next we remark the assumption $p>2$ is enough to show that $s_{f, q}$ factors through $K_{q}^{M}(A)$. In fact, by the compatibility of $s_{f, q}$ with the product structure of the Milnor $K$-group, the problem again reduces to the case $q=2$. So Chapter I Proposition (3.2) in [9] implies the desired property. We do not need the $q$-th divided power $J^{[q]}$ or $\mathcal{S}_{n}(q)$ with $q>2$ in [9] to see this.

We go back to the proof of Proposition A1.3. We have

$$
\begin{aligned}
s_{f, q} & \circ E_{q}\left(a X \cdot \frac{d T_{1}}{T_{1}} \wedge \ldots \wedge \frac{d T_{q-1}}{T_{q-1}}\right) \\
& =\left(f_{1}-1\right) \log \exp \left(\sum_{n=0}^{\infty} f_{1}^{n}\right)(a X) \frac{d T_{1}}{T_{1}} \wedge \ldots \wedge \frac{d T_{q-1}}{T_{q-1}} \\
& =-a X \cdot \frac{d T_{1}}{T_{1}} \wedge \ldots \wedge \frac{d T_{q-1}}{T_{q-1}} .
\end{aligned}
$$

This means that $s_{f, q} \circ E_{q}=-i d$. Thus, $E_{q}$ is injective.

On the other hand, by [2] (4.2) and (4.3), we have a surjective homomorphism

$$
\rho_{i}: \hat{\Omega}_{B}^{q-1} \oplus \hat{\Omega}_{B}^{q-2} \longrightarrow U_{X}^{i} K_{q}^{M}(B)^{\wedge} / U_{X}^{i+1} K_{q}^{M}(B)^{\wedge}
$$


such that

$$
\begin{aligned}
& \rho_{i}\left(a \frac{d b_{1}}{b_{1}} \wedge \ldots \wedge \frac{d b_{q-1}}{b_{q-1}}, 0\right)=\left\{1+X^{i} a, b_{1}, \ldots, b_{q-1}\right\} \\
& \rho_{i}\left(0, a \frac{d b_{1}}{b_{1}} \wedge \ldots \wedge \frac{d b_{q-2}}{b_{q-2}}\right)=\left\{1+X^{i} a, b_{1}, \ldots, b_{q-2}, X\right\}
\end{aligned}
$$

where $a \in B$, and $b_{i} \in B^{\times}$. This shows that $U_{X}^{i} K_{q}^{M}(B)^{\wedge} / U_{X}^{i+1} K_{q}^{M}(B)^{\wedge}$ is generated by the elements of the form $\left\{1+a X^{i}, T_{1}, \ldots, T_{q-1}\right\}$ where $a \in B$, and $T_{1}, \ldots, T_{q-1}$ are in $I_{B}$. Hence, $E_{q}$ is surjective, which completes the proof.

\section{A.2. Smooth local rings over a ramified base.}

A.2.1. In this section, we fix a ring $B$ as in $\S 1$, and study a ring

$$
A=B / \Im \quad \text { where } \quad \Im=\left(X^{e}-p u\right) .
$$

Here, $u$ is a unit of $B$, and $\Im$ is the ideal of $B$ generated by $X^{e}-p u$. We put $\varphi=X^{e}-p u$. We denote by

$$
\psi: B \longrightarrow A
$$

the canonical homomorphism. (For example, if $A$ is a complete discrete valuation ring with mixed characteristics, one can take $\Lambda$ as in $\S A 1$ such that $A / \Lambda$ is finite and totally ramified. Suppose that $R=\Lambda, B=R[[X]]$, and $f(X)=X^{e}-p u\left(u \in B^{\times}\right)$is the minimal polynomial of a prime element of $A$ over $\Lambda$. Then, $A \simeq B /\left(X^{e}-p u\right)$ and the above condition on $A$ is satisfied.)

Let $D$ be the divided power envelope of $B$ with respect to the ideal $\Im$, namely $D=B\left[\varphi^{r} / r !: r>0\right]$. We also denote by

$$
\psi: D \longrightarrow A
$$

the canonical homomorphism which is the extension of $\psi: B \longrightarrow A$.

We define $J=\operatorname{Ker}(D \stackrel{\psi}{\longrightarrow} A)$. Then, the endomorphism $f$ of $B$ naturally extends to $D$. Since $\varphi=X^{e}-p u$, we have $D=B\left[\varphi^{r} / r !: r>0\right]=$ $B\left[X^{e r} / r !: r>0\right]$. Hence, $f(J) \subset p D$ holds. So $f_{1}=p^{-1} f: J \longrightarrow D$ can be defined. Since $f\left(\hat{\Omega}_{B}^{q-1}\right) \subset p^{q-1} \hat{\Omega}_{B}^{q-1}, f_{q-1}=p^{-(q-1)} f$ can be defined on $\hat{\Omega}_{B}^{q-1}$, and

$$
f_{q}=\frac{1}{p^{q}} f: J \otimes \hat{\Omega}_{B}^{q-1} \longrightarrow D \otimes \hat{\Omega}_{B}^{q-1}
$$

can be also defined. 
A.2.2. Recall that $B=R[[X]]$. We denote the image of $X$ in $A$ by the same letter $X$. For $i>0$, we define $U_{X}^{i} A^{\times}=1+X^{i} A$. We note that $U_{X}^{1} A^{\times}$ is $p$-adically complete.

Lemma A.2.1. Let $e^{\prime}=[e /(p-1)]+1$ be the smallest integer $i$ such that $i \geq e /(p-1)+1$. Then, $x \mapsto x^{p}$ gives an isomorphism

$$
U_{X}^{e^{\prime}} A^{\times} \simeq U_{X}^{e+e^{\prime}} A^{\times} .
$$

The proof is standard (cf. [19] Chap.V Lemme 2).

For an element $x \in U_{X}^{e+e^{\prime}} A^{\times}$, by Lemma A2.1, there is a unique $y \in$ $U_{X}^{e^{\prime}} A^{\times}$such that $y^{p}=x$. We denote this element by $x^{1 / p}$. By the same way, for $x \in U_{X}^{n e+e^{\prime}} A^{\times}$, a unique element $y \in U_{X}^{e^{\prime}} A^{\times}$such that $y^{p^{n}}=x$, is denoted by $x^{1 / p^{n}}$. Since $U_{X}^{1} A^{\times}$is a $\mathbf{Z}_{p}$-module, we also use the notation $x^{1 / m p^{n}}$ for an integer $m$ with $(p, m)=1$ for $x \in U_{X}^{n e+e^{\prime}} A^{\times}$.

Recall that $D=B\left[\varphi^{r} / r !: r>0\right]=B\left[X^{e r} / r !: r>0\right]$. Let $U_{X}^{1} D$ be the ideal of $D$ generated by all $X^{e r} / r$ ! with $r>0$. We define a homomorphism

$$
E_{1, A}: U_{X}^{1} D \longrightarrow A^{\times}
$$

by

$$
E_{1, A}\left(a X^{e r} / r !\right)=\psi\left(E_{1}\left(a X^{e r}\right)\right)^{1 / r !}
$$

where $a \in B$, and $E_{1}: X B \longrightarrow B^{\times}$is the map defined in $\S$ A.1. Since $e r \geq e \operatorname{ord}_{p}(r !)+e^{\prime}, \psi\left(E_{1}\left(a X^{e r}\right)\right)^{1 / r !}$ is well-defined by the above remark.

A.2.3. For $q>0$, we will define $E_{q, A}$ similarly as in 1.3. For $i>1$, let $U_{X}^{1}\left(D \otimes \hat{\Omega}_{B}^{q-1}\right)$ (resp. $\left.U_{X}^{i}\left(D \otimes \hat{\Omega}_{B}^{q-1}\right)\right)$ be the subgroup generated by $a \cdot\left(d T_{1}\right) / T_{1} \wedge \ldots \wedge\left(d T_{q-1}\right) / T_{q-1}$ where $a \in U_{X}^{1} D$ (resp. $a \in X^{i} D$ ) and $T_{j} \in I_{B}$.

Let $K_{q}^{M}(A)$ be the $q$-th Milnor $K$-group. As in 1.3 , for $i \geq 0$, let $U_{X}^{i} K_{q}^{M}(A)$ be the subgroup generated by $\left\{1+X^{i} A, A^{\times}, \ldots, A^{\times}\right\}$and $\{1+$ $\left.X^{i} A, A^{\times}, \ldots, A^{\times}, X\right\}$, and define $K_{q}^{M}(A)^{\wedge}$ to be the completion of $K_{q}^{M}(A)$ with respect to the filtration $U_{X}^{i} K_{q}^{M}(A)$. We denote by $U_{X}^{i} K_{q}^{M}(A)^{\wedge}$ the closure of $U_{X}^{i} K_{q}^{M}(A)$ in $K_{q}^{M}(A)^{\wedge}$. Note that $U_{X}^{1} K_{q}^{M}(A)^{\wedge}$ is $p$-adically complete, namely $U_{X}^{1} K_{q}^{M}(A)^{\wedge}=\lim _{\leftarrow} U_{X}^{1} K_{q}^{M}(A) / p^{n}$. We also note that by definition, a natural homomorphism $K_{q}^{M}(B)^{\wedge} \longrightarrow K_{q}^{M}(A)^{\wedge}$ exists.

We define

$$
E_{q, A}: U_{X}^{1}\left(D \otimes \hat{\Omega}_{B}^{q-1}\right) \longrightarrow K_{q}^{M}(A)^{\wedge}
$$

by

$$
E_{q, A}\left(a \cdot \frac{d T_{1}}{T_{1}} \wedge \ldots \wedge \frac{d T_{q-1}}{T_{q-1}}\right)=\left\{E_{1, A}(a), \psi\left(T_{1}\right), \ldots, \psi\left(T_{q-1}\right)\right\}
$$

where $T_{i} \in I_{B}$. 
Recall that $f_{q}=p^{-q} f: J \otimes \hat{\Omega}_{B}^{q-1} \longrightarrow D \otimes \hat{\Omega}_{B}^{q-1}$ is defined (cf. (19)). We put

$$
S_{f}^{q}(A, B)=D \otimes \hat{\Omega}_{B}^{q-1} /\left(d\left(D \otimes \hat{\Omega}_{B}^{q-2}\right)+\left(f_{q}-1\right)\left(J \otimes \hat{\Omega}_{B}^{q-1}\right)\right) .
$$

The filtration on $D \otimes \hat{\Omega}_{B}^{q-1}$ induces a filtration on $S_{f}^{q}(A, B)$, which we denote by $U_{X}^{i} S_{f}^{q}(A, B)$. Our aim in this section is to prove

Theorem A.2.2. $E_{q, A}$ induces an isomorphism

$$
E_{q, A}: U_{X}^{1} S_{f}^{q}(A, B) \stackrel{\simeq}{\longrightarrow} U_{X}^{1} K_{q}^{M}(A)^{\wedge}
$$

which preserves the filtrations.

A.2.4. Let $\hat{\Omega}_{A}^{q-1}$ be the $p$-adic completion of $\Omega_{A}^{q-1}$. We begin with the following lemma.

Lemma A.2.3. There exists a homomorphism

$$
\exp _{p^{2}}: \hat{\Omega}_{A}^{q-1} \longrightarrow K_{q}^{M}(A)^{\wedge}
$$

such that

$$
a \frac{d b_{1}}{b_{1}} \wedge \ldots \wedge \frac{d b_{q-1}}{b_{q-1}} \mapsto\left\{\exp \left(p^{2} a\right), b_{1}, \ldots, b_{q-1}\right\}
$$

where $a \in A, b_{i} \in A^{\times}$, and $\exp (x)=\Sigma_{n \geq 0} x^{n} / n$ !. Furthermore, $d \hat{\Omega}_{A}^{q-2}$ is contained in the kernel of $\exp _{p^{2}}$.

Proof. By Corollary 2.5 in [14], we have a homomorphism

$$
\exp _{p^{2}, B}: \hat{\Omega}_{B}^{q-1} / d \hat{\Omega}_{B}^{q-2} \longrightarrow K_{q}^{M}(B)^{\wedge}
$$

such that

$$
a \frac{d b_{1}}{b_{1}} \wedge \ldots \wedge \frac{d b_{q-1}}{b_{q-1}} \mapsto\left\{\exp \left(p^{2} a\right), b_{1}, \ldots, b_{q-1}\right\}
$$

where $a \in B$ and $b_{i} \in B^{\times}$. Consider the exact sequence

$$
\Im \otimes_{B} \hat{\Omega}_{B}^{q-1} \longrightarrow \hat{\Omega}_{B}^{q-1} / d \hat{\Omega}_{B}^{q-2} \longrightarrow \hat{\Omega}_{A}^{q-1} / d \hat{\Omega}_{A}^{q-2} \longrightarrow 0 .
$$

Since $\Im \otimes \hat{\Omega}_{B}^{q-1}$ is clearly in the kernel of $\psi \circ \exp _{p^{2}, B}: \hat{\Omega}_{B}^{q-1} / d \hat{\Omega}_{B}^{q-2} \longrightarrow$ $K_{q}^{M}(B)^{\wedge} \longrightarrow K_{q}^{M}(A)^{\wedge}$, the map $\exp _{p^{2}, B}$ induces the desired homomorphism

$$
\exp _{p^{2}}: \hat{\Omega}_{A}^{q-1} / d \hat{\Omega}_{A}^{q-2} \longrightarrow K_{q}^{M}(A)^{\wedge}
$$

Corollary A.2.4. For any $a \in A \backslash\{0\}$, in $K_{2}(A)^{\wedge}$ we have

(1) $\left\{\exp \left(p^{2} a\right), a\right\}=0$

(2) $\left\{\exp \left(p^{3} a\right), p\right\}=0$. 
Proof. In the proof of Lemma 2.5 in [12], we showed that

$$
\left\{\exp \left(p^{2} a\right), a\right\}+\left\{\exp \left(p^{2} b\right), b\right\}=\left\{\exp \left(p^{2}(a+b)\right), a+b\right\} .
$$

This formula holds for $a, b$ such that $a, b, a+b \in A \backslash\{0\}$, hence (1) is reduced to the case $a \in A^{\times}$, so follows from Lemma A2.3.

The equation (2) is a consequence of (1). In fact,

$$
\left\{\exp \left(p^{3} a\right), a\right\}=p\left\{\exp \left(p^{2} a\right), a\right\}=0
$$

by (1). So we have

$$
\left\{\exp \left(p^{3} a\right), p\right\}=\left\{\exp \left(p^{3} a\right), a p\right\} .
$$

But the latter is zero again by (1).

\section{A.2.5. We first show}

Lemma A.2.5. $E_{q, A}$ vanishes on $d\left(U_{X}^{1}\left(D \otimes \hat{\Omega}_{B}^{q-2}\right)\right)$.

As in Lemma A1.2, it suffices to show $E_{2, A}\left(d\left(a X^{e r} / r !\right)\right)=0$ for $a \in$ $D$ and $r$ such that $f(a)=a^{p}$ and $r \geq p$. Let $E^{A H}(T)=\Pi_{(p, m)=1}(1-$ $\left.T^{i}\right)^{-\mu(m) / m} \in \mathbf{Z}_{p}[[T]]$ be the Artin-Hasse exponential. We have

$$
\begin{aligned}
& E_{2, A}\left(d\left(a X^{e r} / r !\right)\right) \\
& =\left\{\psi\left(E^{A H}\left(a X^{e r}\right)\right)^{1 /(r-1) !}, \psi\left(X^{e}\right)\right\}+\left\{\psi\left(E^{A H}\left(a X^{e r}\right)\right)^{1 / r !}, \psi(a)\right\} \\
& =\left\{E^{A H}\left((p \psi(u))^{r} \psi(a)\right)^{1 /(r-1) !}, p \psi(u)\right\}+\left\{E^{A H}\left((p \psi(u))^{r} \psi(a)\right)^{1 / r !}, \psi(a)\right\} \\
& =\left\{E^{A H}\left((p \psi(u))^{r} \psi(a)\right)^{1 /(r-1) !}, p\right\}+\left\{E^{A H}\left((p \psi(u))^{r} \psi(a)\right)^{1 / r !}, \psi\left(a u^{r}\right)\right\} .
\end{aligned}
$$

Hence, Lemma A2.5 is reduced to show the following.

Lemma A.2.6. For any $a \in A \backslash\{0\}$,

(1) $\left\{E^{A H}\left(p^{r} a\right)^{1 /(r-1) !}, p\right\}=0$,

(2) $\left\{E^{A H}\left(p^{r} a\right)^{1 / r !}, a\right\}=0$.

Proof of Lemma A2.6. From $E^{A H}(T)=\Sigma_{m=0}^{\infty} \exp \left(T^{p^{m}} / p^{m}\right)$, in order to prove Lemma A2.6 (1), it is enough to show $\left\{\exp \left(\left(p^{r} a\right)^{p^{m}} / p^{m}(r-1) !\right), p\right\}=$ 0 , which follows from Corollary A2.4 (2). By the same method, Lemma A2.6 (2) follows from Corollary A2.4 (1).

By Lemma A2.5, $E_{q, A}$ induces a homomorphism

$$
\begin{aligned}
E_{q, A}: & U_{X}^{1}\left(D \otimes \hat{\Omega}_{B}^{q-1}\right) / d\left(U_{X}^{1}\left(D \otimes \hat{\Omega}_{B}^{q-2}\right)\right) \\
& =U_{X}^{1}\left(D \otimes \hat{\Omega}_{B}^{q-1}\right) /\left[U_{X}^{1}\left(D \otimes \hat{\Omega}_{B}^{q-1}\right) \cap d\left(D \otimes \hat{\Omega}_{B}^{q-2}\right)\right] \longrightarrow K_{q}^{M}(A)^{\wedge} .
\end{aligned}
$$


A.2.6. Next we have to show that $E_{q, A}$ vanishes on

$$
\begin{gathered}
\operatorname{Image}\left(J \otimes \hat{\Omega}_{B}^{q-1} \stackrel{f_{q}-1}{\longrightarrow} D \otimes \hat{\Omega}_{B}^{q-1} \longrightarrow D \otimes \hat{\Omega}_{B}^{q-1} / d\left(D \otimes \hat{\Omega}_{B}^{q-2}\right)\right) \\
\cap U_{X}^{1}\left(D \otimes \hat{\Omega}_{B}^{q-1}\right) / d\left(U_{X}^{1}\left(D \otimes \hat{\Omega}_{B}^{q-2}\right)\right) .
\end{gathered}
$$

We assume that

$$
\varpi=\sum_{r>0} \frac{\left(X^{e}-p u\right)^{r}}{r !}\left(\eta_{r}+X \omega_{r}\right)
$$

satisfies

$$
\left(f_{q}-1\right)(\varpi) \in U_{X}^{1}\left(D \otimes \hat{\Omega}_{B}^{q-1}\right) \quad\left(\bmod d\left(D \otimes \hat{\Omega}_{B}^{q-2}\right)\right)
$$

where $\eta_{r} \in \hat{\Omega}_{R}^{q-1}$ and $\omega_{r} \in \hat{\Omega}_{B}^{q-1}$. We write $u=u_{0}+u_{1} X$ with $u_{0} \in R^{\times}$ and $u_{1} \in B$. We put

$$
\eta=\frac{1}{p} \sum_{r>0} \frac{\left(-p u_{0}\right)^{r}}{r !} \eta_{r}
$$

So $\varpi-p \eta \in U_{X}^{1}\left(D \otimes \hat{\Omega}_{B}^{q-1}\right)$. The assumption (23) implies that

$$
\left(f_{q}-1\right)(p \eta) \in d\left(\hat{\Omega}_{R}^{q-2}\right) .
$$

Lemma A.2.7. $\eta$ can be written as $\eta=p \eta_{1}$ such that $\eta_{1} \in d\left(\hat{\Omega}_{R}^{q-2}\right)$.

By the standard method, Lemma A2.7 is obtained from (24) (cf. [6] Chap.0 Corollaire 2.3.14).

We put

$$
\varpi_{1}=\sum_{r>0} \frac{\left(X^{e}-p u\right)^{r}-\left(-p u_{0}\right)^{r}}{r !} \eta_{r}, \quad \varpi_{2}=\sum_{r>0} \frac{\left(X^{e}-p u\right)^{r}}{r !} X \omega_{r} .
$$

So by $(22)$,

$$
\varpi=\varpi_{1}+\varpi_{2}+p \eta
$$

From (24) and the definition of $E_{q, A}$, we have

$$
E_{q, A}\left(\left(f_{q}-1\right) \varpi\right)=E_{q, A}\left(\left(f_{q}-1\right) \varpi_{1}\right)+E_{q, A}\left(\left(f_{q}-1\right) \varpi_{2}\right) .
$$

If we write $\omega_{r}=\Sigma_{i} a_{r, i}\left(d T_{r, i, 1}\right) / T_{r, i, 1} \wedge \ldots \wedge\left(d T_{r, i, q-1}\right) / T_{r, i, q-1}$ with $T_{r, i, j} \in$ $I_{B}$, by the definition of $E_{q, A}$,

$$
\begin{aligned}
E_{q, A}\left(\left(f_{q}-1\right) \varpi_{2}\right) & =\sum_{r, i}\left\{\psi\left(\exp \left(\frac{\left(X^{e}-p u\right)^{r}}{r !} X a_{r, i}\right)\right), \psi\left(T_{r, i, 1}\right), \ldots, \psi\left(T_{r, i, q-1}\right)\right\} \\
& =0 .
\end{aligned}
$$

Next we calculate $E_{q, A}\left(\left(f_{q}-1\right) \varpi_{1}\right)$. We write $\eta_{r}=\Sigma_{i} b_{r, i}\left(d T_{r, i, 1}\right) / T_{r, i, 1} \wedge$ $\ldots \wedge\left(d T_{r, i, q-1}\right) / T_{r, i, q-1}$ with $T_{r, i, j} \in I_{B}$. Then by the definition of $E_{q, A}$, we 
get

$$
\begin{aligned}
& E_{q, A}\left(\left(f_{q}-1\right) \varpi_{1}\right) \\
& =\sum_{r, i}\left\{\psi \exp \left(\frac{\left(X^{e}-p u\right)^{r}-\left(-p u_{0}\right)^{r}}{r !} b_{r, i}\right), \psi\left(T_{r, i, 1}\right), \ldots, \psi\left(T_{r, i, q-1}\right)\right\} \\
& =\sum_{r, \imath}\left\{\psi \exp \left(\frac{-\left(-p u_{0}\right)^{r}}{r !} b_{r, i}\right), \psi\left(T_{r, i, 1}\right), \ldots, \psi\left(T_{r, i, q-1}\right)\right\} .
\end{aligned}
$$

By Lemma A2.7,

$$
\sum_{r>0} \frac{\left(-p u_{0}\right)^{r}}{r !} \eta_{r}=p \eta=p^{2} \eta_{1}
$$

and $\eta_{1} \in d\left(\hat{\Omega}_{R}^{q-2}\right)$. Hence by Lemma A2.3,

$$
E_{q, A}\left(\left(f_{q}-1\right) \varpi_{1}\right)=-\exp _{p^{2}}\left(\eta_{1}\right)=0 \text {. }
$$

This shows that

$$
\begin{gathered}
\operatorname{Image}\left(J \otimes \hat{\Omega}_{B}^{q-1} \stackrel{f_{q}-1}{\longrightarrow} D \otimes \hat{\Omega}_{B}^{q-1} \longrightarrow D \otimes \hat{\Omega}_{B}^{q-1} / d\left(D \otimes \hat{\Omega}_{B}^{q-2}\right)\right) \\
\cap\left[U_{X}^{1}\left(D \otimes \hat{\Omega}_{B}^{q-1}\right) / d\left(U_{X}^{1}\left(D \otimes \hat{\Omega}_{B}^{q-2}\right)\right)\right]
\end{gathered}
$$

is in the kernel of $E_{q, A}$. Namely, $E_{q, A}$ induces a homomorphism

$$
E_{q, A}: U_{X}^{1} S_{f}^{q}(A, B) \longrightarrow U_{X}^{1} K_{q}^{M}(A)^{\wedge}
$$

A.2.7. As in $\S 1, E_{q, A}$ has the inverse. The target group of the symbol map in [9] has two components as in $\S 1$, and its projection to the first component is

which satisfies

$$
s_{f, q, A}: K_{q}^{M}(A) \longrightarrow S_{f}^{q}(A, B)
$$

$$
\begin{aligned}
& s_{f, q, A}\left(\left\{a_{1}, \ldots, a_{q}\right\}\right) \\
& \quad=\sum_{i=1}^{q}(-1)^{i-1} \frac{1}{p} \log \frac{f\left(\widetilde{a}_{i}\right)}{\widetilde{a}_{i}^{p}} \frac{d \widetilde{a}_{1}}{\widetilde{a}_{1}} \wedge \ldots \wedge \frac{d \widetilde{a}_{i-1}}{\widetilde{a}_{i-1}} \wedge \frac{f}{p}\left(\frac{d \widetilde{a}_{i+1}}{\widetilde{a}_{i+1}}\right) \wedge \ldots \wedge \frac{f}{p}\left(\frac{d \widetilde{a}_{q}}{\widetilde{a}_{q}}\right)
\end{aligned}
$$

where $\tilde{a}_{i}$ is an element of $D^{\times}$such that $\psi\left(\widetilde{a}_{i}\right)=a_{i}$. This does not depend on the choices of $\widetilde{a}_{i}$.

As in $\S 1, s_{f, q, A} \circ E_{q, A}=-i d$ because if $T_{1}, \ldots, T_{q-1}$ are in $I_{B}$, then

$$
s_{f, q}\left(\left\{a, \psi\left(T_{1}\right), \ldots, \psi\left(T_{q-1}\right)\right\}\right)=\frac{1}{p} \log \frac{f(\widetilde{a})}{\widetilde{a}^{p}} \frac{d T_{1}}{T_{1}} \wedge \ldots \wedge \frac{d T_{q-1}}{T_{q-1}} .
$$

(As we note in $\S \mathrm{A} 1$, we are allowed to take $T_{i}=X$ using our convention.) So $E_{q}$ is injective. On the other hand, by considering $U_{X}^{i} K_{q}^{M}(A)^{\wedge} /$ $U_{X}^{i+1} K_{q}^{M}(A)^{\wedge}$ as in $\S \mathrm{A} 1$, we know that $E_{q}$ is surjective. Hence, $E_{q}$ is bijective. It is clear that $E_{q}$ preserves the filtrations. This completes the proof of Theorem A2.2. 
Remark A.2.8. By a slight modification, we can deal with more general ring, for example, a ring of the form $R\left[X_{1}, \ldots, X_{r}\right]_{\left(m_{R}, X_{1}, \ldots, X_{r}\right)} /\left(X_{1}^{e_{1}} \cdot \ldots\right.$. $\left.X_{r}^{e_{r}}-p u\right)$ by the same method.

Remark A.2.9. Assume that \#I $\leq q-2$. Then we have $U_{X}^{1} S_{f}^{q}(A, B)=$ $S_{f}^{q}(A, B)$ and $U_{X}^{1} K_{q}^{M}(A)^{\wedge}=K_{q}^{M}(A)^{\wedge}$. So Theorem 1.1 says that we have an isomorphism

$$
E_{q, A}: S_{f}^{q}(A, B) \simeq K_{q}^{M}(A)^{\wedge} .
$$

This isomorphism in this special case was obtained in Kato [10].

Corollary A.2.10. There exists a homomorphism

$$
\exp _{p}: \Omega_{A}^{q-1} \longrightarrow K_{q}^{M}(A)^{\wedge}
$$

such that

$$
a \frac{d b_{1}}{b_{1}} \wedge \ldots \wedge \frac{d b_{q-1}}{b_{q-1}} \mapsto\left\{\exp (p a), b_{1}, \ldots, b_{q-1}\right\}
$$

where $a \in A$ and $b_{i} \in A^{\times}$.

Proof. By [2] Lemma 4.2, it suffices to show

$$
\sum_{i=1}^{l}\left\{\exp \left(p a_{i}\right), a_{i}, b_{1}, \ldots, b_{q-2}\right\}=\sum_{i=1}^{m}\left\{\exp \left(p a_{i}^{\prime}\right), a_{i}^{\prime}, b_{1}, \ldots, b_{q-2}\right\}
$$

for $a_{i}, a_{i}^{\prime}, b_{j} \in A^{\times}$such that $\Sigma a_{i}=\Sigma a_{i}^{\prime}$. So we may assume $q=2$.

Let $s_{f, 2, A}$ be the map as above. Then, for $a \in A^{\times}$, taking $\widetilde{a} \in B$ such that $\psi(\widetilde{a})=a$, we can calculate

$$
\begin{aligned}
s_{f, 2}\{\exp (p a), a\}= & \left(f_{1}-1\right) \log \exp (p \widetilde{a}) f_{1}(d \log \widetilde{a}) \\
& -\frac{1}{p} \log \left(f(\widetilde{a}) / \widetilde{a}^{p}\right) d \log \exp (p \widetilde{a}) \\
= & f_{1}(d \widetilde{a})-p d \widetilde{a} .
\end{aligned}
$$

Here, we used $d\left(\log \left(f(\widetilde{a}) / \widetilde{a}^{p}\right) \cdot \widetilde{a}\right)=0$ in $S_{f}^{q}(A, B)$. The final equation is clearly additive in $a$. Since

$$
s_{f, 2, A}: U_{X}^{1} K_{2}(A)^{\wedge} \longrightarrow U_{X}^{1} S_{f}^{2}(A, B)
$$

is bijective by Theorem $\mathrm{A} 2.2,\{\exp (p a), a\}$ is additive in $a$, and (27) is satisfied. Hence, $\exp _{p}$ is a homomorphism.

Remark A.2.11. One has $\left\{\exp \left(p \widetilde{a} \widetilde{b} X^{i}\right), \widetilde{b} X^{i}\right\}=\left\{\exp \left(p \widetilde{a}\left(1+\widetilde{b} X^{i}\right)\right)\right.$, $\left.\left(1+\widetilde{b} X^{i}\right)\right\}$ for $i>0, \widetilde{a} \in B$, and $\widetilde{b} \in B^{\times}$(note that $\left\{\exp \left(p \widetilde{a} \widetilde{b} X^{i}\right), \widetilde{b} X^{i}\right\}$ makes sense in $K_{2}(B)^{\wedge}$ by our convention (cf.1.3)) because $s_{f, 2}\left(\left\{\exp \left(p \widetilde{a} \tilde{b} X^{i}\right), \widetilde{b} X^{i}\right\}\right)$ 
$=s_{f, 2}\left(\left\{\exp \left(p \tilde{a}\left(1+\widetilde{b} X^{i}\right)\right),\left(1+\widetilde{b} X^{i}\right)\right\}\right)$ (cf. remark after the definition of $s_{f, q}$ in the proof of Proposition A1.3). Hence, we have

$$
\exp _{p}\left(a d b_{1} X^{i} \wedge \frac{d b_{2}}{b_{2}} \wedge \ldots \wedge \frac{d b_{q-1}}{b_{q-1}}\right)=\left\{\exp \left(p a b_{1} X^{i}\right), b_{1} X^{i}, b_{2}, \ldots, b_{q-1}\right\}
$$

for $a \in A, b_{1}, \ldots, b_{q-1} \in A^{\times}$, and $i>0$.

Remark A.2.12. Theorem 1.1 gives a different proof of the main result in [13]. Let $K$ be a complete discrete valuation field with integer ring $O_{K}$, and $X$ be a smooth scheme over $O_{K}$. We denote by $i: Y \longrightarrow X$ the special fiber of $X$ and by $j: X_{\eta} \longrightarrow X$ the generic fiber of $X$.

Let $K_{q}^{M}\left(O_{X}\right)$ be the sheaf of Milnor $K$-groups, and $\mathcal{S}_{n}(r)$ be the syntomic complex on $D\left(Y_{e t}\right)$ defined in [9] for $n>0$. Then by using (a modified version of ) Theorem A2.2, we can show the existence of an isomorphism

$$
i^{*} K_{q}^{M}\left(O_{X}\right) / p^{n} \simeq \mathcal{H}^{q}\left(\mathcal{S}_{n}(q)\right)
$$

for $q$ such that $0<q<p-1$. In fact, Theorem A2.2 says that $U^{1} i^{*} K_{q}^{M}\left(O_{X}\right) / p^{n} \simeq U^{1} \mathcal{H}^{q}\left(\mathcal{S}_{n}(q)\right)$. (Here $U^{1} i^{*} K_{q}^{M}\left(O_{X}\right)$ is defined similarly as above.)

Comparing this isomorphism (28) with a result in [2], we have an exact sequence

$$
0 \longrightarrow \mathcal{H}^{q}\left(\mathcal{S}_{n}(q)\right) \longrightarrow i^{*} R^{q} j_{*} \mathbf{Z} / p^{n}(q) \longrightarrow W_{n} \Omega_{Y, \log }^{q-1} \longrightarrow 0 .
$$

This exact sequence was proved in [13] by a different method.

For $r$ such that $q<r<p-1$, we can also prove the bijectivity of

$$
\left(i^{*} K_{q}^{M}\left(O_{X}\right) / p\right) \otimes \mu_{p}^{\otimes(r-q)} \simeq \mathcal{H}^{q}\left(\mathcal{S}_{1}(r)\right)
$$

by a similar method as in this paper. Then by (29), (30), and the theory of Fontaine and Messing, we can show the existence of a distinguished triangle

$$
\mathcal{S}_{n}(r) \longrightarrow \tau_{\leq r} i^{*} R j_{*} \mathbf{Z} / p^{n}(r) \longrightarrow W_{n} \Omega_{Y, \log }^{r-1}[-r]
$$

which was the main theorem of [13]. Tsuji extended this result to much more general setting [20] [21], and proved the semi-stable conjecture by Fontaine and Jannsen.

(*) Note. This paper was written long time ago, but the author still thinks the problems to determine all $\operatorname{gr}^{i} K_{q}^{M}(K)$ for complete discrete valuation fields of mixed characteristics, and also to determine the kernels of the exponential homomorphisms for Milnor $K$-groups (cf. [15]) are interesting problems. 


\section{References}

[1] S. BLoch, Algebraic K-theory and crystalline cohomology. Publ. Math. IHES 47 (1977), 187-268.

[2] S. BlOCh, K. KATO, p-adic etale cohomology. Publ. Math. IHES 63 (1986), 107-152.

[3] M. Demazure, Lectures on p-divisible groups. Lecture Notes in Math. 302, Springer (1972).

[4] J.-M. Fontaine, W. Messing, p-adic periods and p-adic étale cohomology. Contemporary Math. 67 (1987), 179-207.

[5] J. Graham, Continuous symbols on fields of formal power series, Algebraic K-theory II. Lecture Notes in Math. 342, Springer-Verlag (1973), 474-486.

[6] L. Illusie, Complexes de de Rham Witt et cohomologie crystalline. Ann. Sci. Éc. Norm. Super. $4^{e}$ série t. 12 (1979), 501-661.

[7] K. KATO, Residue homomorphisms in Milnor K-theory, in Galois groups and their representations. Adv. St. in Pure Math. 2 (1983), 153-172.

[8] K. KATO, A generalization of local class field theory by using K-groups I. J. Fac. Sci. Univ. Tokyo 26 (1979), 303-376, II, ibid 27 (1980), 603-683, III, ibid 29 (1982), 31-43.

[9] K. KATo, On p-adic vanishing cycles (applications of ideas of Fontaine-Messing). Adv. St. in Pure Math. 10 (1987), 207-251.

[10] K. Kato, The explicit reciprocity law and the cohomology of Fontaine-Messing. Bull. Soc. Math. France 119 (1991), 397-441.

[11] M. Kolster, $K_{2}$ of non-commutative local rings. J. Algebra 95 (1985), 173-200.

[12] M. KuRIhARA, On two types of complete discrete valuation fields. Compos. Math. 63 (1987), 237-257.

[13] M. Kurihara, A note on p-adic etale cohomology. Proc. Japan Acad. Ser. A 63 (1987), 275-278.

[14] M. KURIHARA, Abelian extensions of an absolutely unramified local field with general residue field. Invent. math. 93 (1988), 451-480.

[15] M. KURIHARA, The exponential homomorphisms for the Milnor K-groups and an explicit reciprocity law. J. reine angew. Math. 498 (1998), 201-221.

[16] J. NAKAMURA, On the structures of the Milnor K-groups of some complete discrete valuation fields. K-Theory 19 (2000), 269-309.

[17] J. NAKAMURA, On the Milnor K-groups of complete discrete valuation fields. Doc. Math. 5 (2000), 151-200 (electronic).

[18] A.N. PARshin, Class field theory and algebraic K-theory. Uspekhi Mat. Nauk. 30 no 1 (1975), 253-254, (English transl. in Russian Math. Surveys).

[19] J.-P. Serre, Corps locaux (3 ${ }^{e}$ édition), Hermann, Paris, (1968).

[20] T. TsuJI, Syntomic complexes and p-adic vanishing cycles. J. reine angew. Math. 472 (1996), 69-138.

[21] T. TsuJI, p-adic étale cohomology and crystalline cohomology in the semi-stable reduction case. Invent. math. 137 (1999), 233-411.

[22] W. VAN DeR KAllen, The $K_{2}$ of rings with many units. Ann. Sci. Éc. Norm. Sup. $4^{e}$ série t. 10 (1977), 473-515.

[23] S.V. Vostokov, Explicit form of the law of reciprocity. Izv. Acad. Nauk. SSSR 13 (1979), 557-588.

[24] I. ZhuKov, Milnor and topological K-groups of multidimensional complete fields. St. Petersburg Math. J. 9 (1998), 69-105.

Masato KURIHARA

Department of Mathematics,

Tokyo Metropolitan University,

Hachioji, Tokyo, 192-03, Japan

E-mail : m-kuri@comp.metro-u.ac.jp 